\title{
An obligate cell-intrinsic function for CD28 in Tregs
}

\author{
Ruan Zhang, ${ }^{1}$ Alexandria Huynh, ${ }^{1}$ Gregory Whitcher, ${ }^{1}$ JiHoon Chang, ${ }^{1}$ \\ Jonathan S. Maltzman, ${ }^{2}$ and Laurence A. Turka ${ }^{1}$
}

\begin{abstract}
${ }^{1}$ Transplant Institute, Department of Medicine, Beth Israel Deaconess Medical Center, Harvard Medical School, Boston, Massachusetts, USA. 2Department of Medicine, Perelman School of Medicine, University of Pennsylvania, Philadelphia, Pennsylvania, USA.
\end{abstract}

\begin{abstract}
Tregs expressing the transcription factor FOXP3 are critical for immune homeostasis. The costimulatory molecule CD28 is required for optimal activation and function of naive $\mathrm{T}$ cells; however, its role in Treg function has been difficult to dissect, as CD28 is required for thymic Treg development, and blockade of CD28-ligand interactions has confounding effects in trans on nonregulatory cells. To address this question, we created Treg-specific $C d 28$ conditional knockout mice. Despite the presence of normal numbers of FOXP3 ${ }^{+}$cells, these animals accumulated large numbers of activated $T$ cells, developed severe autoimmunity that primarily affected the skin and lungs, and failed to appropriately resolve induced experimental allergic encephalomyelitis. This in vivo functional impairment was accompanied by dampened expression of CTLA-4, PD-1, and CCR6. Disease occurrence was not due to subversion of $\mathrm{Cd28}$-deficient Tregs into pathogenic cells, as complementation with normal Tregs prevented disease occurrence. Interestingly, in these "competitive" environments, $\mathrm{Cd} 28$-deficient Tregs exhibited a pronounced proliferative/survival disadvantage. These data demonstrate clear postmaturational roles for $\mathrm{CD} 28$ in $\mathrm{FOXP3}^{+}$Tregs and provide mechanisms which we believe to be novel to explain how interruption of CD28-ligand interactions may enhance immune responses independent of effects on thymic development or on other cell types.
\end{abstract}

\section{Introduction}

Tregs are a central element in the maintenance of self-tolerance. The transcription factor forkhead box p3 (FOXP3) is a key molecule mediating the development and function of natural Tregs (nTregs) (1-3). Patients with IPEX and scurfy mice, each of which lack functional FOXP3, have a severe systemic autoimmune syndrome characterized by a lack of functional Tregs and multiorgan disease $(4,5)$. Moreover, induced loss of $\mathrm{FOXP}^{+} \mathrm{T}$ cells in healthy adult animals leads to rapid onset of catastrophic autoimmunity (6), further demonstrating the importance of FOXP3 ${ }^{+}$Tregs in immune homeostasis.

CD28 is the prototypical and best-characterized costimulatory molecule on T cells $(7,8)$. CD28 signals are critical for optimal naive $\mathrm{T}$ cell activation, cytokine production, proliferation, and survival. Consistent with this, in rodent models of transplantation, transient blockade of the CD28 ligands CD80 and CD86 using CTLA4Ig leads to apoptosis of alloantigen-reactive cells, induction of Tregs, and long-term allograft survival $(9,10)$. However, perturbation of this system may have undesired immunostimulatory effects. CD28 is required for the intrathymic generation of nTregs. Thus, mice deficient in CD28 or its ligands have a dramatically reduced number of nTregs and develop accelerated autoimmunity on an NOD background (11). Moreover, there are also circumstances in which CTLA4Ig enhances immune responses. Blockade of CD28 engagement by CTLA4Ig leads to a rapid decrease of Tregs both in the thymus and in the periphery $(11,12)$ and, possibly as a direct result, breaks self-tolerance or

Conflict of interest: Laurence A. Turka has a family member employed by Novartis and an equity position in Novartis, and his family receives royalties from Myriad Genetics. Jonathan S. Maltzman has a family member employed by Morphotek and an equity position in GlaxoSmithKline.

Citation for this article: J Clin Invest. 2013;123(2):580-593. doi:10.1172/JCI65013. transplantation-tolerance in models in which Tregs play a major role in maintaining those states $(13,14)$.

The mechanisms for these effects remain incompletely defined. Previous studies addressing the role of CD28 in Tregs have used either $\mathrm{Cd} 28^{-/-}$mice or blocking anti-B7 antibodies and/or CTLA4Ig. This body of work, while demonstrating the importance of CD28 in Tregs, has a number of limitations. First, as CD28 is required for intrathymic Treg development $(11,15)$, it is difficult to unravel the role of CD28 in Treg function and maintenance in these animals. Alternative approaches, such as the use of anti-B7 or CTLA4Ig, have the confounding variables of blocking both CD28 and CTLA-4 signals and doing so on all cells, not just Tregs. Thus, the experimental models may be confounded by the trans effects of loss of CD28-mediated costimulation and cytokine production by effector T cells or by interruption of CTLA-4 binding to CD80 and CD86, with the resultant loss of CTLA-4 mediated negative signals on effector T cells or CTLA-4-mediated suppression by Tregs $(16,17)$. Understanding the role of CD28 in Tregs is of particular clinical importance given the recent results of the phase III study of belatacept (an enhanced affinity variant of CTLA4Ig) showing higher rates, and more severe grades, of rejection (albeit with similar 1-year graft survival) in the belatacept-treated groups compared with a CNI-treated group (18).

To define the role of CD28 in the homeostasis and function of $\mathrm{FOXP}^{+}$Tregs, we generated CD28-conditional knockout mice (Cd28- $\Delta$ Treg mice) that target CD28 in FOXP3 ${ }^{+}$Tregs. We found that loss of CD28 in Tregs leads to two distinct and separable abnormalities - cell-intrinsic proliferation/survival defects, which manifest under competitive conditions, and functional impairment in vivo, which is accompanied by dampened expression of CTLA-4, PD-1, and CCR6. The net result is an autoimmune disease in $C d 28-\Delta$ Treg mice that is Treg intrinsic and prevented by complementation with CD28-sufficient Tregs. These data demon- 


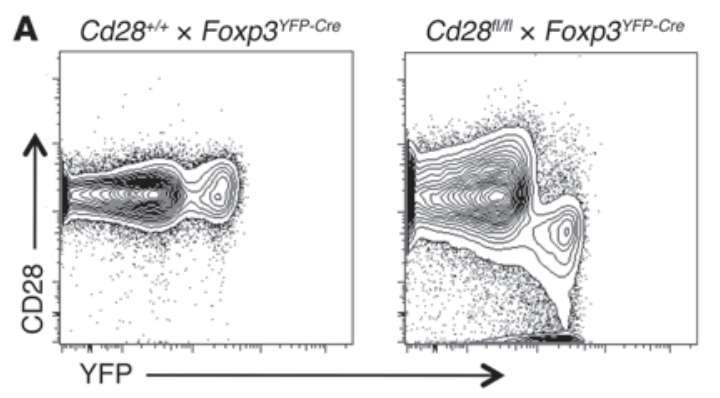

B
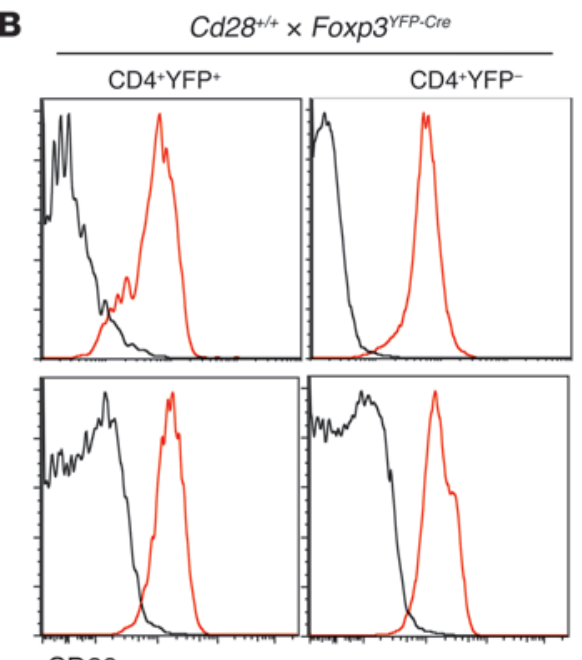

CD28

\section{$\mathrm{Cd} 28^{\text {flM }} \times$ Foxp3 $3^{\text {YFP.Cre }}$}

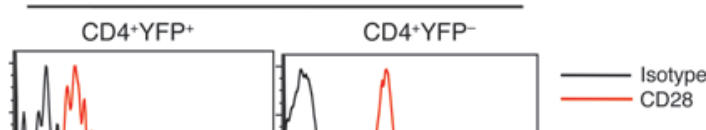

Thymus

\section{Figure 1}

CD28 expression in control and Cd28- $\Delta$ Treg mice. (A) CD28 and YFP expression in gated CD4+ lymph node cells. (B) CD28 expression in the indicated cells from thymus or lymph node. Analyses in $\mathbf{A}$ and $\mathbf{B}$ are from 6-week-old littermates and are representative of 3 independent experiments and litters.

strate clear postmaturational roles for CD28 in FOXP3 ${ }^{+}$Tregs and provide what we believe to be a novel mechanism to explain how interruption of CD28/B7 signaling may enhance immune responses independent of effects on thymic development or on other cell types.

\section{Results}

Generation and characterization of Cd28- $\Delta$ Treg mice. We used BAC recombineering to introduce 2 loxP sites into the intronic sequences of the $\mathrm{Cd} 28$ locus. Together, the inserted loxP sites flanked the extracellular (exon 2) and transmembrane (exon 3) domains of Cd28 as well as some intervening intronic sequences (see Methods and Supplemental Figure 1A; supplemental material available online with this article; doi:10.1172/JCI65013DS1). CD28-floxed mice were genotyped by PCR and Southern blotting (Supplemental Figure 1B), and we confirmed that insertion of the loxP sites did not interfere with the normal expression of the Cd28 gene (Supplemental Figure 1C).

To generate mice with a specific deletion of CD28 in FOXP3 ${ }^{+}$ Tregs, $C d 28^{f l f l}$ mice were bred with Foxp $3^{Y F P-C r e}$ mice (19), and we refer to mice which carry the $C d 28^{f l / f l} C r e^{+}$genotype as $C d 28-\Delta$ Treg mice. Flow cytometric analysis revealed that CD28 was inactivated in peripheral $\mathrm{CD}^{+} \mathrm{YFP}^{+}$Tregs from blood, lymph node, and spleen but not in $\mathrm{YFP}^{-}$"conventional" $\mathrm{CD}^{+} \mathrm{T}$ cells or in $\mathrm{CD}^{+}$ $\mathrm{T}$ cells (Figure 1, A and B, and data not shown). As shown in Figure $1 \mathrm{~A}$, virtually all $\mathrm{YFP}^{+}$cells are CD28-, and, conversely, almost all
$\mathrm{CD}^{-} 8^{-}$cells are $\mathrm{YFP}^{+}$, indicating the fidelity of Cre expression in these mice and the absence of significant leakiness. In the thymus, low levels of CD28 expression were observed on a portion of the $\mathrm{YFP}^{+}$cells in Cd28- $\Delta$ Treg mice (Figure 1B). Consistent with other mouse models that use Foxp $3^{\text {Cre }}$, this is most likely due to the fact that these are newly generated Tregs in which the already translated CD28 protein has not been fully degraded.

The construct that we have used should delete sequences encoding exons 2 and 3, which encode the extracellular and transmembrane portions of the molecule, respectively, and the remaining portions of the gene are not expected to be transcribed or expressed. To confirm the absence of a residual truncated protein (which might have positive or negative signaling effects), we performed Western blotting using an antibody that binds to exon 4, which encodes an intracellular portion of CD28, and is not contained within the targeting region. We found a detectable specific band only in WT Tregs (Supplemental Figure 2). A nonspecific smaller band of approximately $40 \mathrm{kD}$ was present in all samples and is not consistent with a truncated protein, since the undeleted exons (exon 1 and exon 4), even if translated, would lead to a protein predicted to be $<10 \mathrm{kD}$. Thus, $C d 28-\Delta$ Treg mice effectively delete detectable $\mathrm{CD} 28$ protein in peripheral $\mathrm{FOXP}^{+}$cells.

Homeostasis of Tregs in Cd28- $\Delta$ Treg mice. Analysis of young Cd28$\Delta$ Treg mice revealed normal populations of APCs and NK and myeloid cells in thymi, spleens, lymph nodes, and bone marrow 
A $C d 28^{+/+} \times$Foxp3 $3^{\text {YFP-Cre }} \quad C d 28^{\text {th/t }} \times$ Foxp3 $3^{\text {YFP-Cre }}$

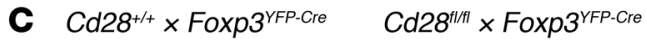
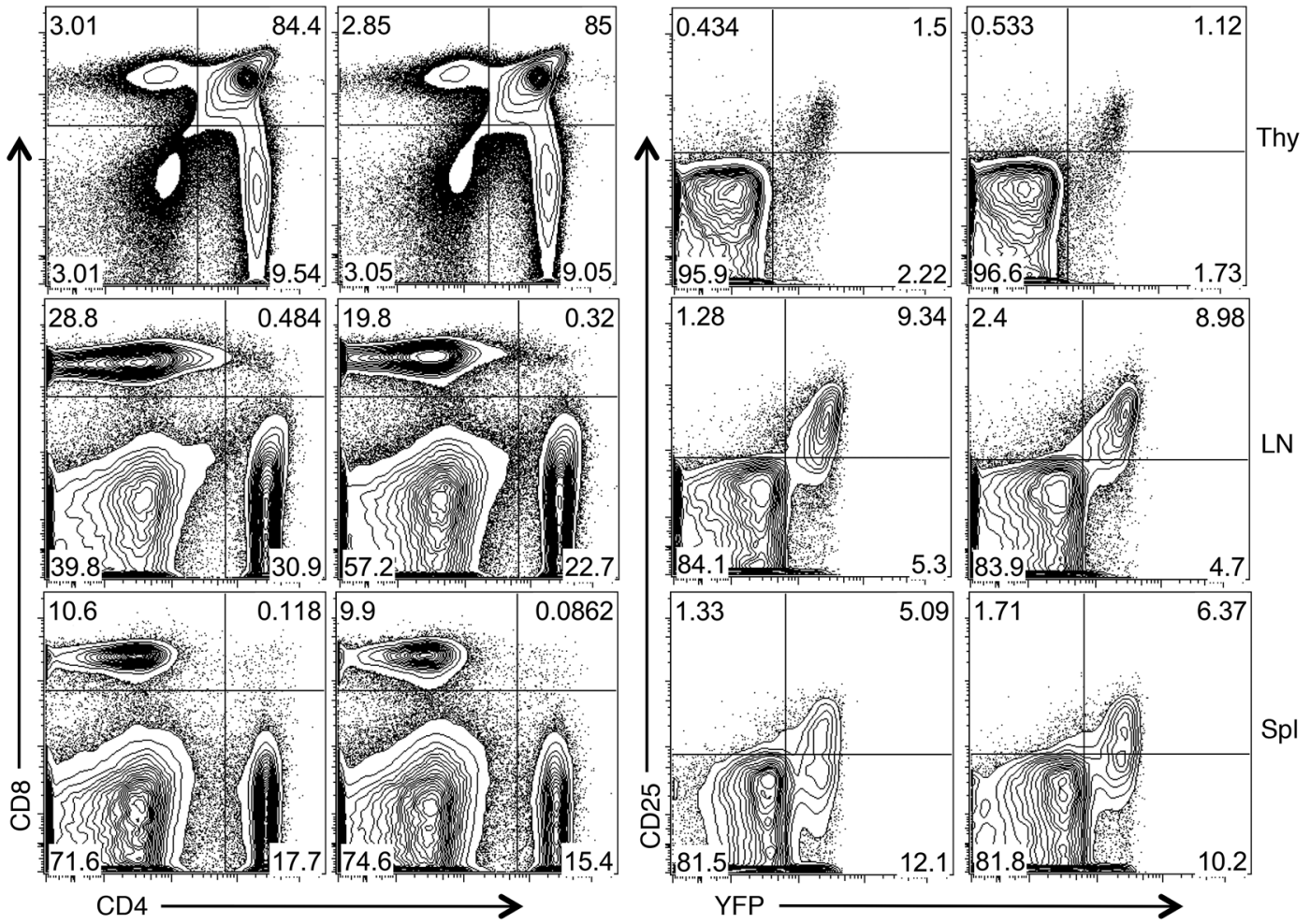

B
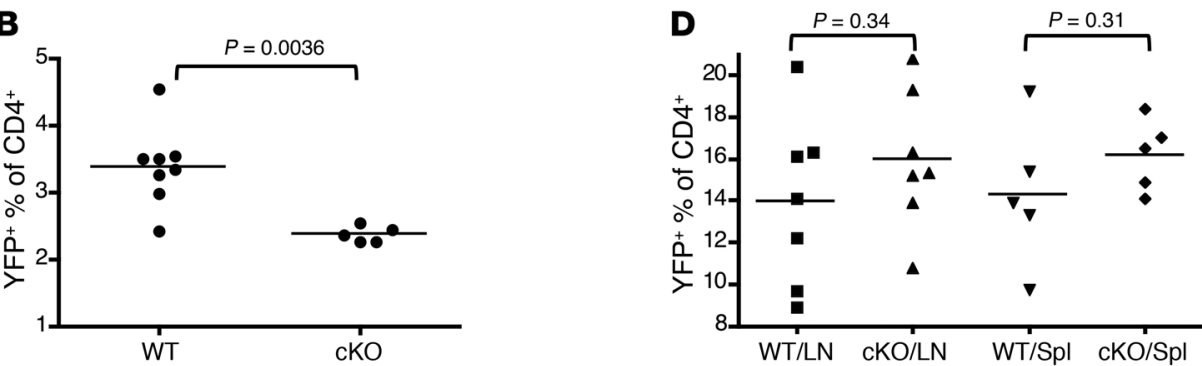

Figure 2

Treg development and homeostasis in Cd28- $\Delta$ Treg mice. (A and B) Representative analysis for (A) CD4 and CD8 and (B) CD25 and YFP in

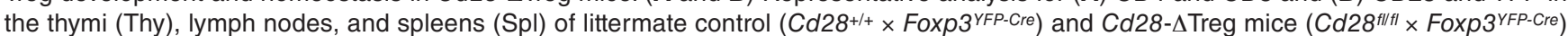
mice (age 5 weeks). (C and D) Percentage of Tregs (defined as YFP+) in CD4+CD8- ${ }^{-}$cells from (C) thymi and (D) lymph nodes or spleens of

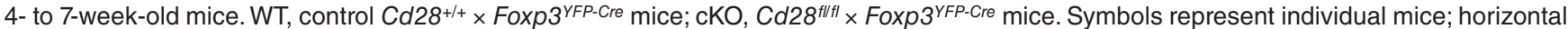
bars indicate the mean.

(Supplemental Figure 3, A-C). Overall T and B cells were normal in all tissues except lymph nodes, in which a roughly $30 \%-50 \%$ increase in B cells was observed (Supplemental Figure 3A), with a reciprocal decrease in $\mathrm{T}$ cells (Figure $2 \mathrm{~A}$ ).

The proportions and total numbers of CD4/8 double-negative, double-positive, and single-positive cells were normal in the thymi of young $C d 28-\Delta$ Treg mice compared with those in littermate controls (Figure 2A and data not shown). However, there was a roughly $25 \%-30 \%$ decrease in the percentage of $\mathrm{FOXP}^{+}$cells among the CD4 single-positive population in the thymi of $C d 28-\Delta$ Treg mice (Figure 2, B and C). Despite this, Treg percentages in lymph nodes and spleens of $C d 28-\Delta$ Treg mice were similar to those in littermate controls (Figure 2, B and D), and the level of CD25 expression on Cd28- $\Delta$ Tregs was comparable to that on WT Tregs in all lymphoid tissues examined (Figure 2B).
To further analyze Treg homeostasis, $C d 28-\Delta$ Treg mice and littermate controls were pulsed with BrdU for 3 days and sacrificed, and $\mathrm{BrdU}^{+}$Tregs were enumerated in lymphoid tissues. Consistent with the observation of reduced thymic Tregs in $C d 28-\Delta$ Treg mice, as shown in Figure 2, B and C, we observed decreased percentages of $\mathrm{BrdU}^{+}$Tregs within the thymi of these animals compared with control WT or male Cd28 ${ }^{f l+} F o x p 3^{Y F P-C r e}$ mice (Figure 3, $\mathrm{A}$ and $\mathrm{B})$. In contrast, the percentage of lymph node and splenic Tregs that incorporated BrdU during the pulse period was similar in control and Cd28- $\Delta$ Treg mice (Figure 3, A and B, and data not shown). To exclude the confounding effects of newly generated Tregs from the thymus, which have been exported to the periphery, we thymectomized mice, pulsed them with BrdU 1 week later, and serially assayed peripheral blood T cells. We found similar rates of disappearance of $\mathrm{BrdU}^{+}$peripheral Tregs, 

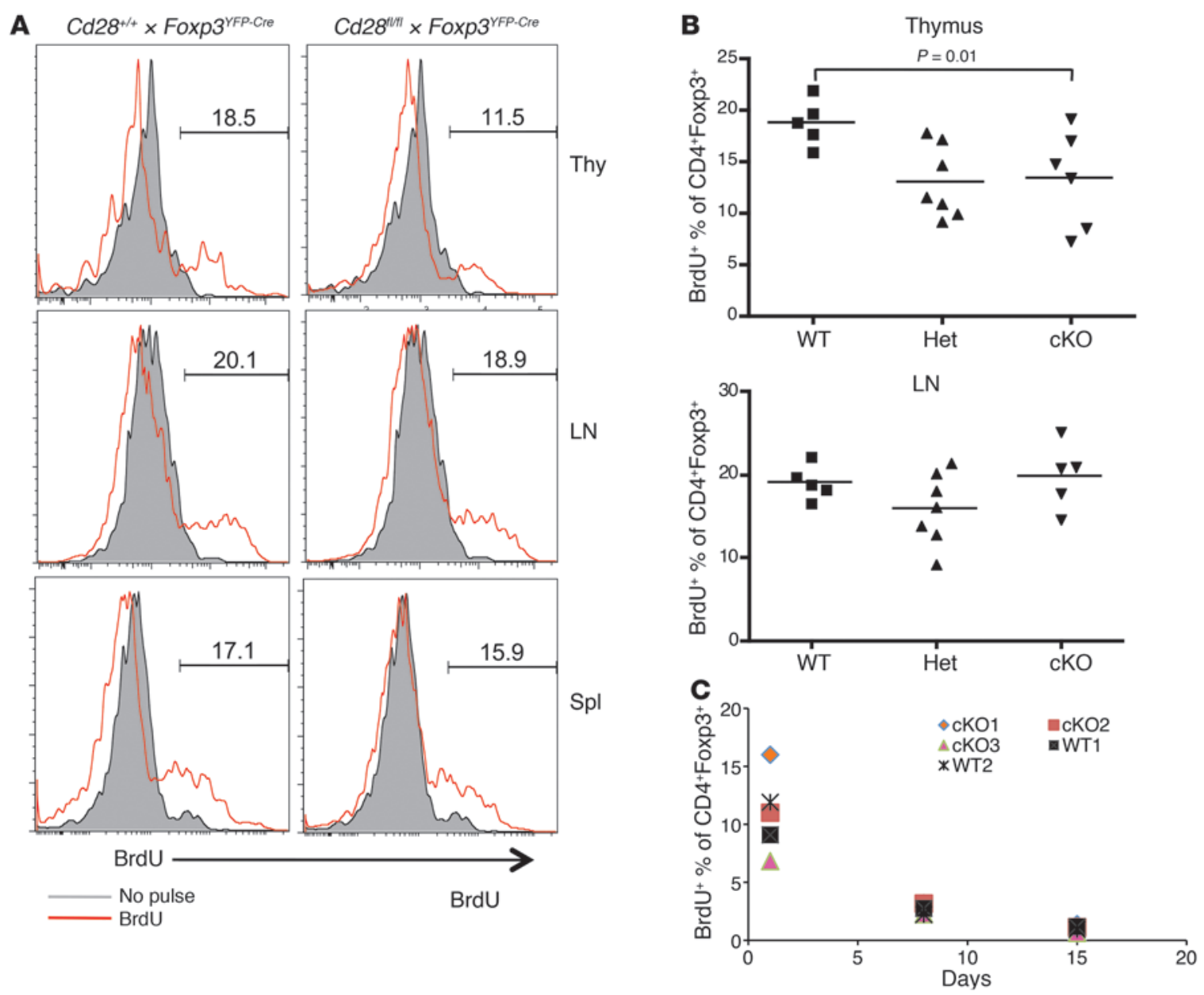

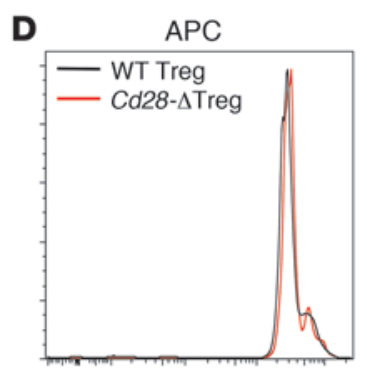

Cell tracer
$\mathrm{APC}+$ anti-CD3

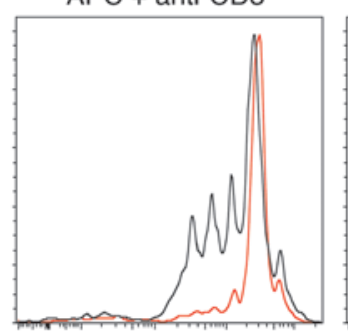

$\ln _{1} \sqrt{h}$

$\mathrm{APC}+$ anti-CD3 + rlL2
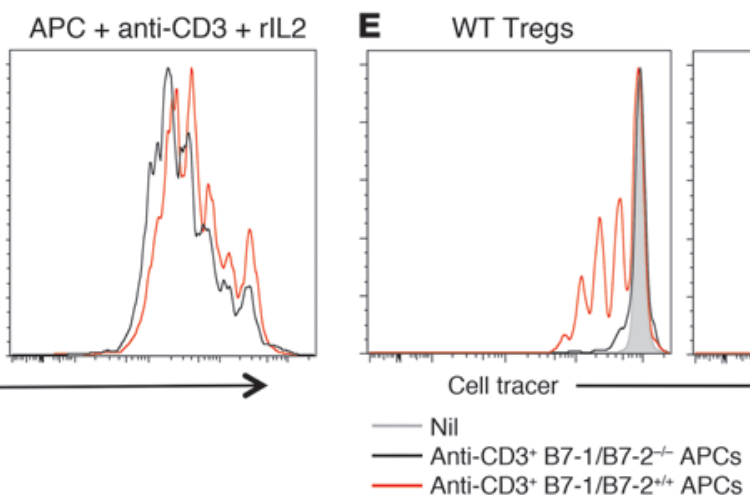

\section{Figure 3}

Treg homeostasis. (A and B) BrdU incorporation assay. 4- to 7-week-old WT or Cd28- $\Delta$ Treg mice were pulsed with BrdU every 12 hours for 3 days and sacrificed, and relevant tissues were analyzed by flow cytometry. Representative BrdU staining of gated CD4 $4^{+}$FOXP3 $3^{+}$cells is shown in $\mathbf{A}$,

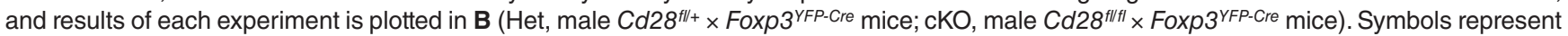
individual mice; horizontal bars indicate the mean. (C) BrdU incorporation in thymectomized mice, as per the protocol in A. CD4+FOXP3 ${ }^{+}$cells from blood were analyzed at the indicated times after the last BrdU pulse. (D and E) Sorted WT Tregs or Cd28- $\Delta$ Tregs were labeled with CellTrace Violet and were stimulated by soluble CD3 plus mitomycin-treated T cell-depleted splenocytes (D) with or without rIL-2 or (E) with B7-1/B7-2 double-knockout T cell-depleted splenocytes.

regardless of whether they expressed CD28 (Figure 3C), which is in agreement with their indistinguishable steady-state levels (Figure 2, B and C). This rapid loss of detectable BrdU-positive cells is likely due to extensive cell division of Tregs, as has been previ- ously seen (20). Collectively, these data suggest that, while thymic numbers of Tregs are reduced in Cd28- $\Delta$ Treg mice, once the cells are exported into the periphery, homeostasis, with respect to cell numbers and turnover, is preserved. 
A

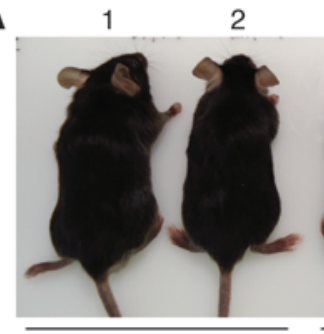

1 month

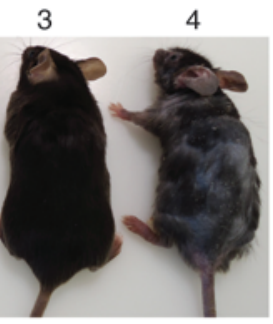

3 month

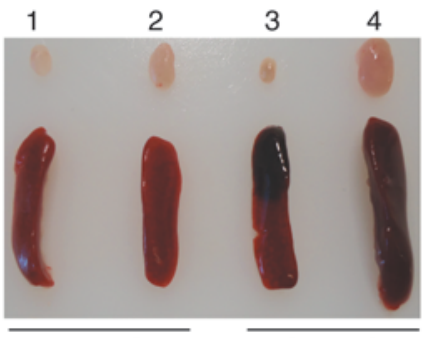

3 month
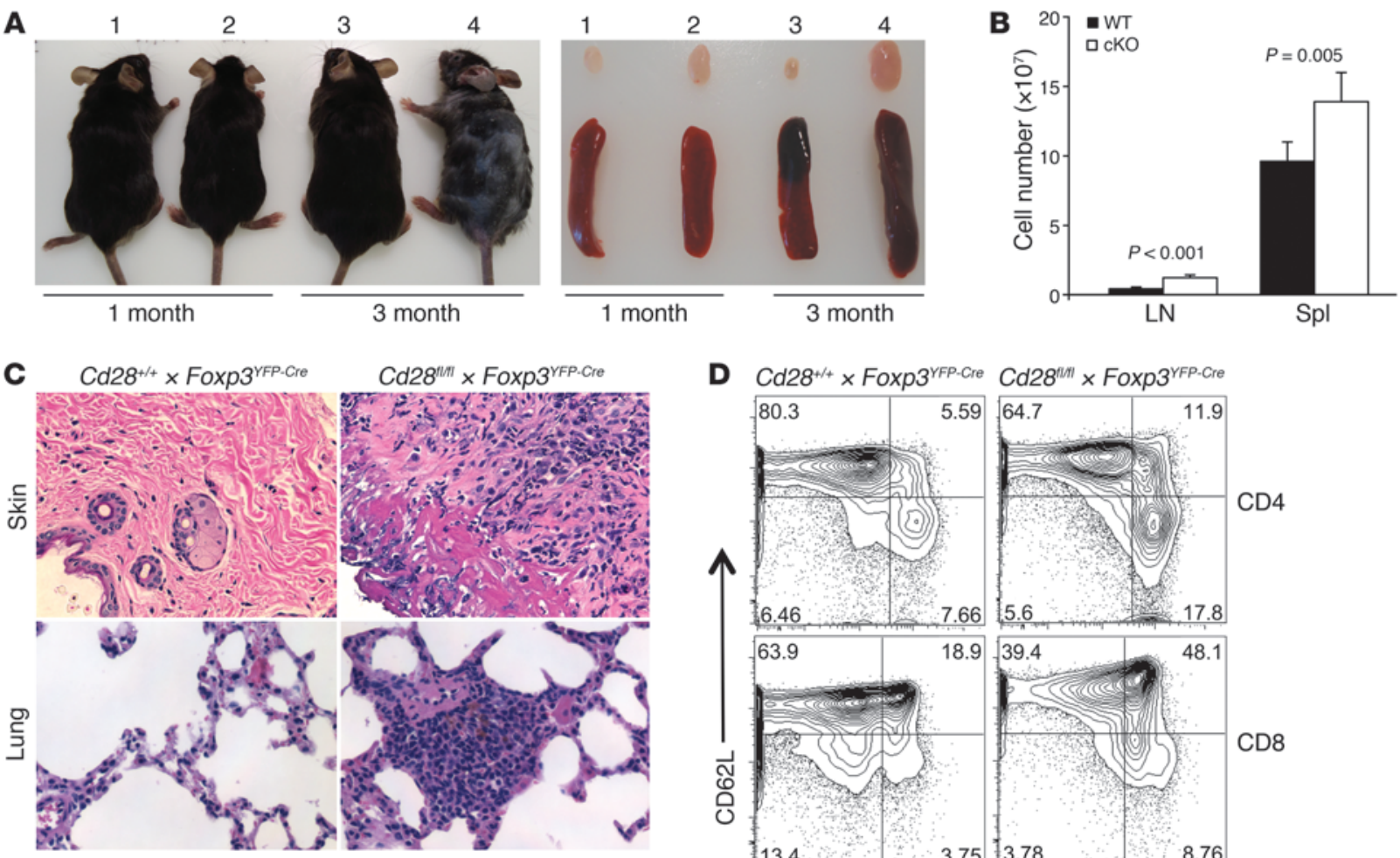

D Cd28 $8^{\text {t+ }} \times$ Foxp $3^{\text {YFP-Cre }}$ Cd $28^{\text {thl }} \times$ Foxp $3^{\text {YFP-Cre }}$
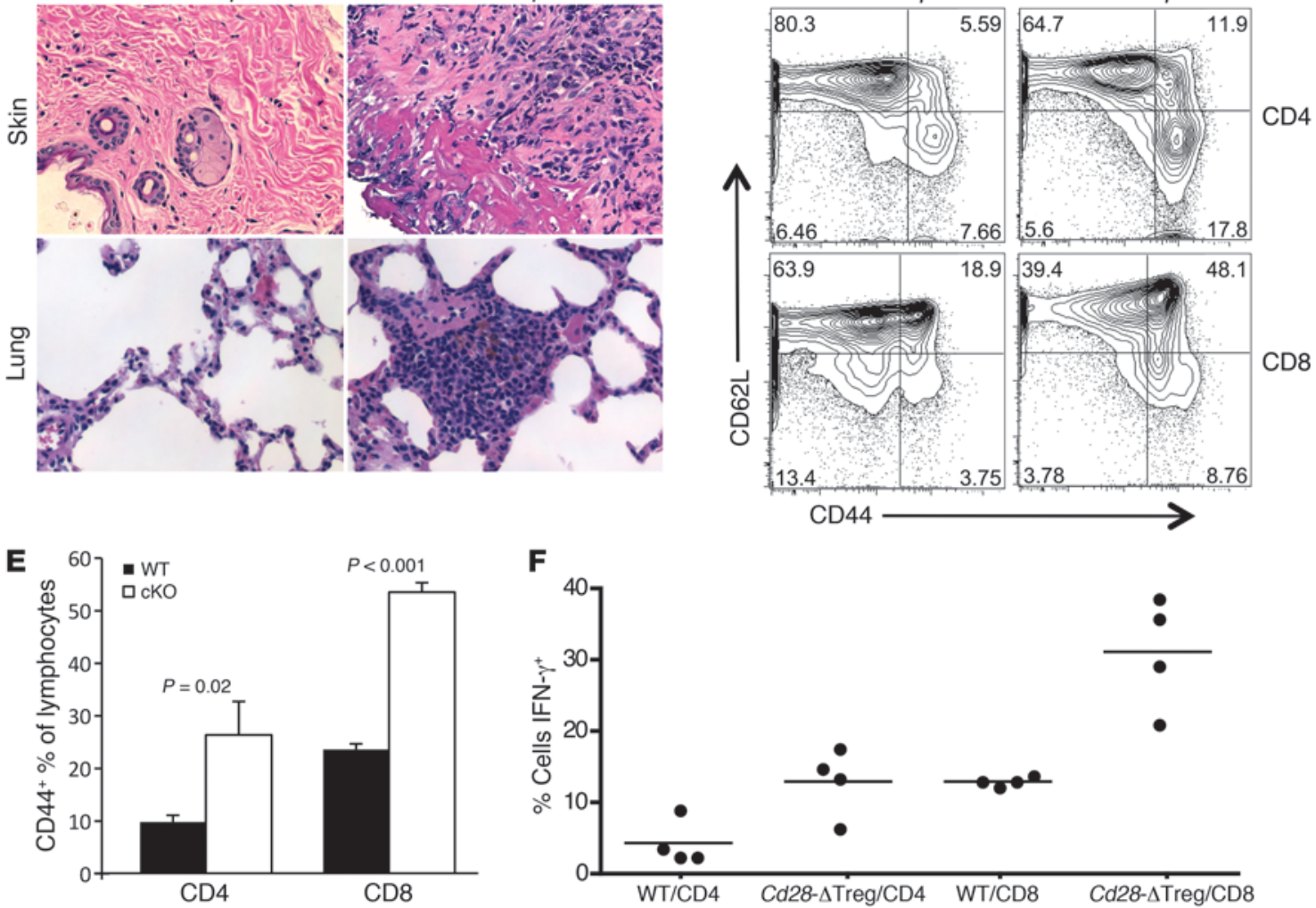

\section{Figure 4}

Cd28- $\Delta$ Treg mice develop autoimmunity with skin inflammation. (A) Gross appearance and lymph nodes and spleens from representative WT littermate control (labeled 1 and 3) and Cd28- $\Delta$ Treg (labeled 2 and 4) mice. (B) Cell numbers in lymph nodes and spleens from 2- to 5-monthold mice. 6 littermate pairs were analyzed. Data represent mean \pm SEM. (C) H\&E staining of skin and lung tissue in a 3-month-old Cd28- $\Delta$ Treg mouse. Original magnification, $\times 400$. (D) CD $44^{+}$CD62L hi cells in lymph nodes of a representative 4-month-old Cd28- $\Delta$ Treg mice. (E) Proportions of $\mathrm{CD} 44^{+}$cells from lymph nodes of 2-month-old mice. 5 littermate pairs were analyzed. Data represent mean $\pm \mathrm{SEM}$. (F) Percentage of IFN- $\gamma^{+}$ cells in stimulated splenocytes from 2- to 5-month-old littermate pairs. Symbols represent individual mice; horizontal bars indicate the mean.

Last, we directly examined the ability of Tregs to proliferate in vitro. Although purified Tregs appear anergic in vitro, TCR, costimulation, and cytokines together can, under appropriate conditions, drive Treg proliferation (21). To examine the role of CD28 costimulation in Treg proliferation, we labeled sorted WT Tregs or Cd28- $\Delta$ Tregs with CellTrace Violet and stimulated them with anti-CD3 mAb in the presence of mitomycin-treated $\mathrm{T}$ cell-depleted splenocytes (Figure 3D). Under these conditions, in contrast to control Tregs, CD28-deficient Tregs proliferated minimally, although this could be "rescued" with exogenous IL-2. It is not currently known what cytokine(s) rIL-2 is "replacing," as neither WT Tregs or Cd28- $\Delta$ Tregs produce IL-2 under these conditions (data not shown). Lack of cell division was not due to cell death, as the viability of $C d 28-\Delta$ Tregs was comparable to that of control Tregs after the 3-day culture (data not shown). Further evidence for the role of CD28 costimulation of Tregs in supporting their proliferation is the fact that no proliferative differences were seen between Tregs from WT mice and those from Cd28- $\Delta$ Treg mice when B7-1/B7-2 double-knockout cells were used as APCs (Figure 3E).

Depletion of CD28 in Tregs leads to autoimmune disease. While appearing normal at birth and through 1 month of age, by 8 to 12 weeks of age $100 \%$ of $C d 28-\Delta$ Treg mice $(n=30)$ developed signs suggestive of autoimmunity (Figure 4A). At onset, animals manifested crusting eyelids with facial hair loss, which progressed to hair loss on the trunk, skin lesions, and an ill appearance characterized 
A

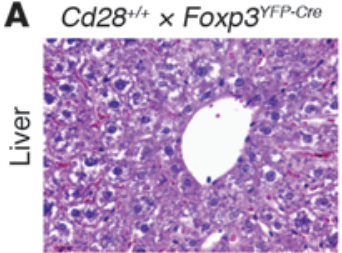

$C d 28^{n t h} \times$ Foxp3 $^{\text {YFP-Cre }}$
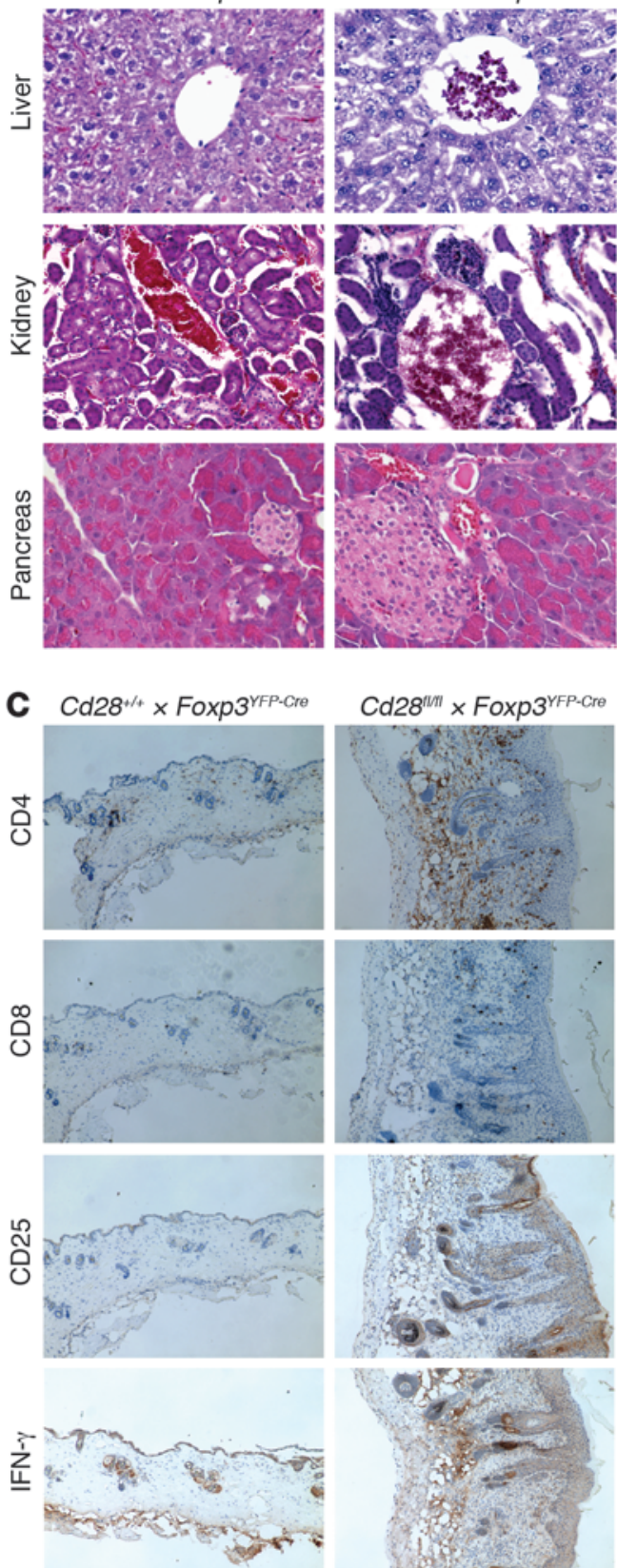

(a)

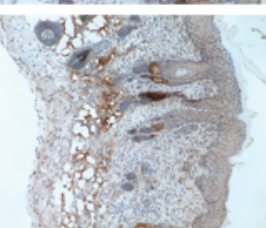

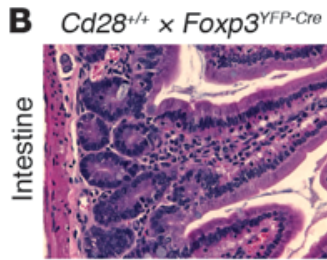
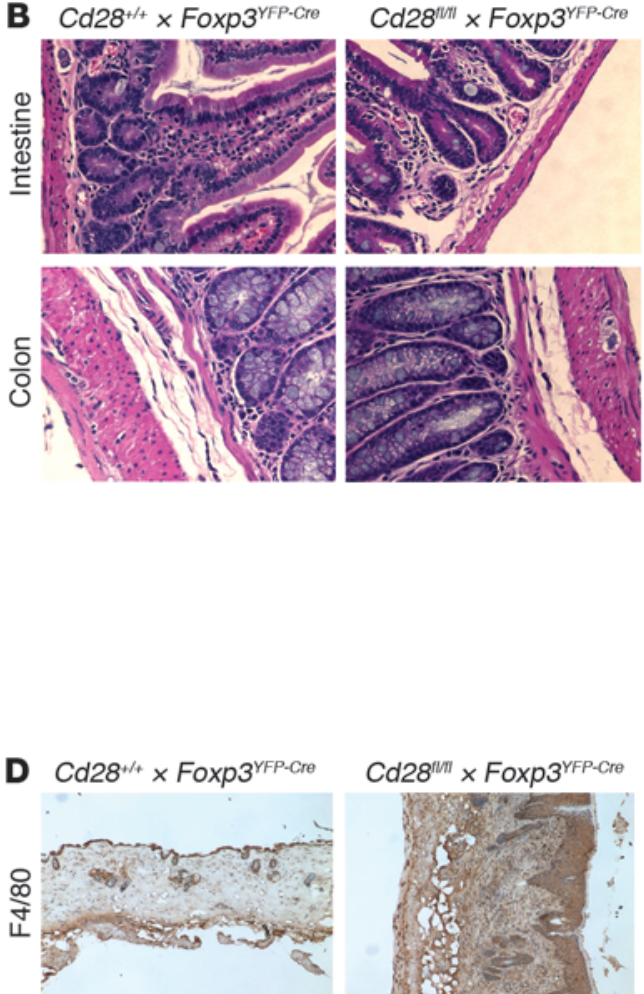
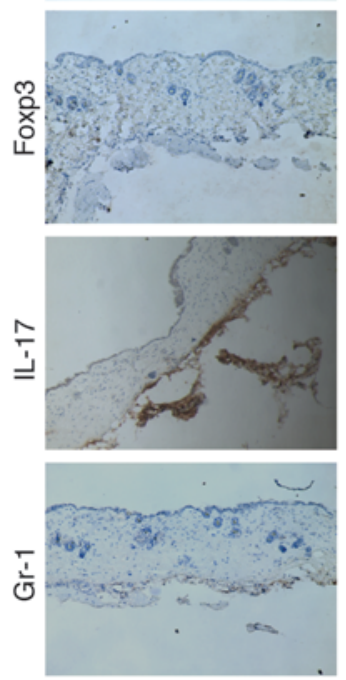

Figure 5

Histologic analysis. (A and B) $H \& E$ staining of $(\mathbf{A})$ livers, kidneys, and pancreata or (B) intestines and colons in 3-month-old Cd28- $\Delta$ Treg mice. (C and D) Immunohistochemistry staining of skin tissues from a 3-monthold Cd28- $\Delta$ Treg mice. Original magnification, $\times 400$ (A and $\mathbf{B})$; $\times 100(\mathbf{C}$ and $\mathbf{D})$. Data are representative of at least 3 animals. by ruffled fur, hunching, and reduced movements in the cage. This was associated with the development of lymphadenopathy and splenomegaly (Figure 4, A and B) and characterized by the accumulation of activated CD $44^{+} \mathrm{T}$ cells (in both the $\mathrm{CD} 4^{+}$and $\mathrm{CD}^{+}$lineages) (Figure 4, D and E) and a high proportion of T cells primed for IFN- $\gamma$ production (Figure $4 \mathrm{~F}$ ). Consistent with the clinical picture of autoimmunity and the presence of high numbers of phenotypically activated $\mathrm{T}$ cells, histological analysis showed marked lymphocytic infiltration of skin and lung and scattered less intense foci of mononuclear cells in liver, pancreas, kidney, intestine, and colon (Figure 4C and Figure 5). Together, these data indicate that the absence of CD28 on Tregs leads to a slowly progressive systemic disease, with some features similar to those of scurfy mice, albeit developing with a slower tempo.
Disease in Cd28- $\Delta$ Treg mice can be prevented by CD28-sufficient Tregs. We considered that the disease observed in $C d 28-\Delta$ Treg mice might be due to lack of normally functioning Tregs. An alternative possibility was that this was an act of commission, not merely omission; i.e., the CD28-deficient Tregs were becoming autoreactive effector cells that were initiating disease. This particularly seemed possible as Tregs are believed to be among the peripheral $T$ cells with the highest affinity for self-antigens (22).

To examine this issue, we first cultured purified (>95\% pure) $\mathrm{CD} 28^{+}$or CD28- Tregs in vitro under proinflammatory conditions and then analyzed them for cytokine production following a brief restimulation. Following culture under Th 1 conditions (Supplemental Figure 4A), 20\%-25\% of viable Tregs (both $\mathrm{CD}_{28}{ }^{+}$or $\mathrm{CD}_{28}^{-}$) lost FOXP3 expression, and none of these 


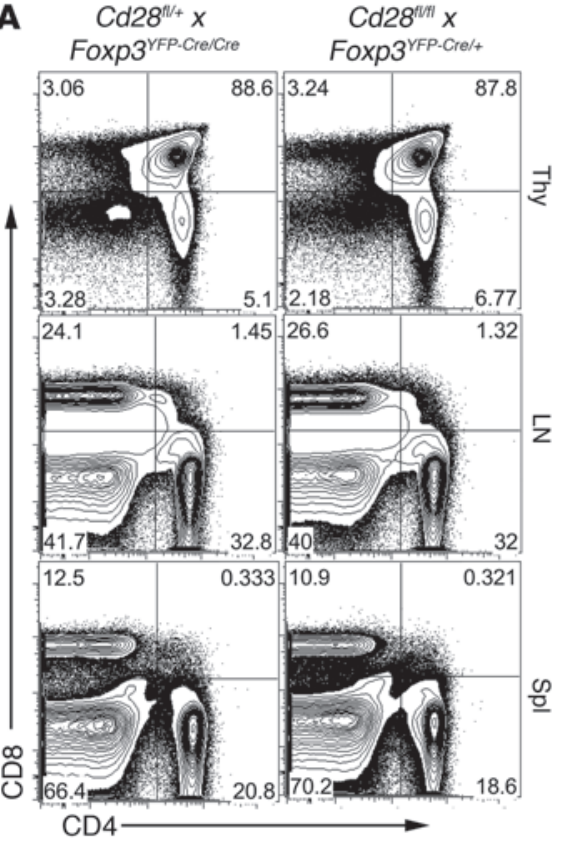

B $\quad C d 28^{n / /+} x \quad C d 28^{n / H / T} x$

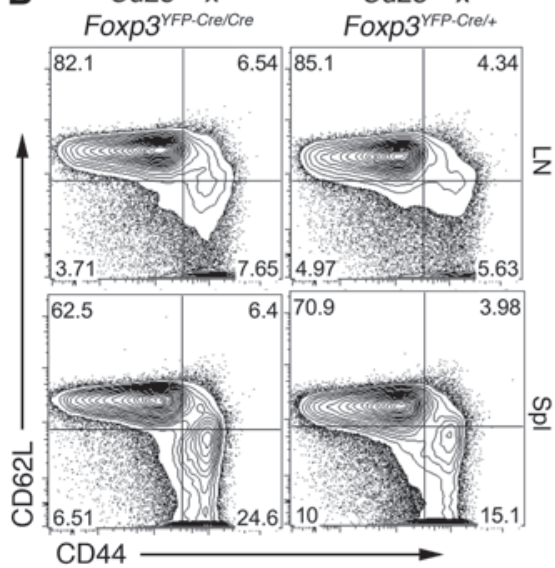

Figure 6

Analysis of female mice heterozygous for Foxp3 ${ }^{Y F P-C r e}$. (A-D) Flow cytometric analyses of thymus, lymph node, and spleen of a representative

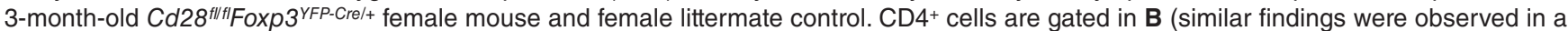
CD8 ${ }^{+}$gate; data not shown) and $\mathbf{C}$, and CD4+FOXP3 ${ }^{+} \mathrm{CD} 25^{+}$cells are gated in $\mathbf{D}$. (E) Percentage of YFP ${ }^{+}$Cd28- $\Delta$ Tregs in a representative $C d 28^{f l / f l}$

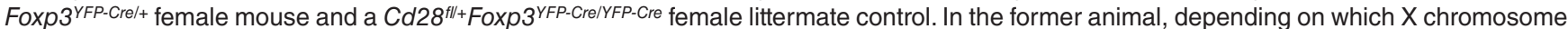
is randomly inactivated, some cells will delete CD28, and others will not. In these mice, YFP+ Tregs are Cd28 $8^{-1-}$, while YFP- Tregs are Cd28+/+. In the control, as the animals are heterozygous for the targeted CD28 allele but homozygous for Foxp3 $3^{\text {YFP-Cre }}$, all Tregs in these mice will be Cd28 ${ }^{+/}$. In both cases, CD4 ${ }^{+}$CD25 ${ }^{+}$FOXP3 ${ }^{+}$lymph node cells are shown. (F) Cd28 flffFoxp $3^{\text {YFP-Cre/ }+}$ female mice were administered BrdU as in Figure 3 , and BrdU staining of $\mathrm{YFP}^{+}$and $\mathrm{YPF}^{-}$cells was separately analyzed.

produced detectable IFN- $\gamma$. Of the cells that retained FOXP3 expression, equivalent percentages $(\sim 25 \%$, i.e., $\sim 15 \%-18 \%$ of the overall number of cells) of CD28 ${ }^{+}$and CD28- Tregs produced IFN- $\gamma$. Under Th17 conditions (Supplemental Figure 4B) a much higher proportion of cells lost FOXP3 expression (and the remaining ones were FOXP3 ${ }^{\mathrm{dim}}$ ) and produced IL-17 as well, but again, no appreciable difference was seen between $\mathrm{CD} 28^{+}$ and CD28- Tregs. 


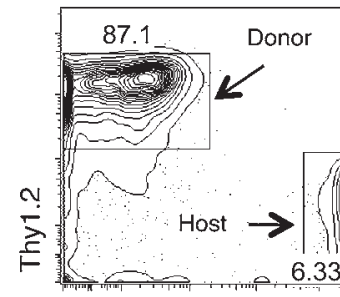

Thy1.1

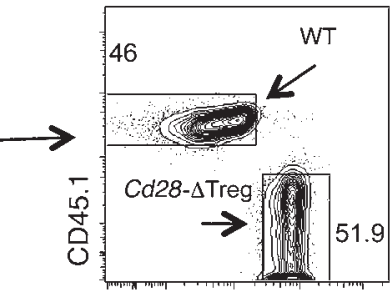

CD45.2

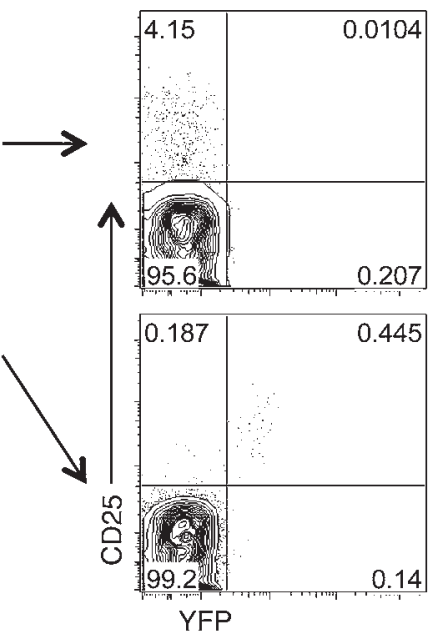

\section{Figure 7}

Cd28- $\Delta$ Treg development in bone marrow chimeras. B6 mice (CD45.2+Thy $1.1^{+}$) were irradiated and reconstituted with $\mathrm{a}$ $1: 1$ ratio of bone marrow cells from WT B6 (CD45.1+Thy $1.2^{+}$) and Cd28- $\Delta$ Treg mice (CD45.2+ Thy $1.2^{+}$). Blood cells were analyzed 6 months later, and $\mathrm{CD} 4^{+}$cells were gated. Note that the WT mice did not carry the Fox-

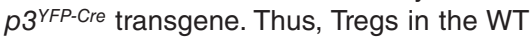
mice are CD25+YFP-, while in the Cd28$\Delta$ Treg mice, they are CD25+YFP+. A total of 7 mice were analyzed with similar results.
To further discriminate whether disease in Cd28- $\Delta$ Treg mice was due to lack of functioning Tregs, we took advantage of the fact that, since foxp 3 is on the $\mathrm{X}$ chromosome, in female mice heterozygous for Foxp $3^{\text {Cre }}$ only approximately half of the Tregs should delete $\mathrm{CD} 28$ due to random inactivation of one $\mathrm{X}$ chromosome. In these mice, $\mathrm{YFP}^{+}$Tregs are $\mathrm{C} d 28^{-1-}$ while $\mathrm{YFP}^{-}$Tregs are $C d 28^{+/+}$. In the female $C d 28^{f /+}{ }^{\circ}$ Foxp $3^{Y F P-C r e / Y F P-C r e}$ littermate control, as the animals are heterozygous for the targeted CD28 allele but homozygous for Foxp $3^{\text {YFP-Cre }}$, all Tregs will be $\mathrm{Cd} 28^{+/-}$.

Three-month-old female Cd28flflFoxp $3^{\text {YFP-Cre/+ }}$ mice (Cre-heterozygous mice) appeared healthy (data not shown). The thymic and peripheral lymphoid compartments had normal percentages of CD4 and CD8 cells, and lymph nodes and spleens were of normal size without an excessive number of CD44 ${ }^{\text {hi }}$ and/or CD62 $\mathrm{L}^{\text {lo }}$ cells (Figure 6, A and B, and data not shown). Interestingly, however, the percentage of Tregs was reduced in both the thymus and the periphery (Figure 6C), as WT Tregs did not undergo a compensatory expansion. Further analysis showed that CD28 was specifically deleted in $\mathrm{YFP}^{+}$(and not $\mathrm{YFP}^{-}$) Tregs and that the percentage of $\mathrm{YFP}^{+}$Tregs in the Cre-heterozygous mice was markedly below 50\% (Figure 6, D and E), which we attribute to the loss of Cd28- $\Delta$ Tregs in a competitive environment. Consistent with this, BrdU labeling revealed that the turnover rate of CD28-sufficient Tregs was approximately twice that of $C d 28-\Delta$ Tregs (Figure $6 \mathrm{~F}$ ). To more specifically test this hypothesis, 1:1 mixtures of WT and Cd28- $\Delta$ Treg bone marrow were used to reconstitute heavily irradiated recipients. Six months after reconstitution, we found that while the two contributed equally to the overall hematopoietic compartment, virtually all Tregs were derived from WT bone marrow (Figure 7).

Suppressive function of $C d 28-\Delta$ Tregs. These data, showing that a complement of normal Tregs is sufficient to prevent disease development in mice harboring CD28-deleted Tregs, suggested that disease was caused by a functional failure of $C d 28-\Delta$ Tregs. Therefore, we next examined the ability of these cells to mediate regulatory responses. As shown in Figure $8 \mathrm{~A}, \mathrm{Cd} 28-\Delta$ Tregs from young healthy mice inhibited the proliferation of naive $T$ cells in an in vitro suppression assay as effectively as did control Tregs.

To examine the function of $C d 28-\Delta$ Tregs in vivo, we used a standard adoptive transfer colitis model in Rag1-/- hosts, wherein mice received $\mathrm{CD}^{+} \mathrm{CD} 25^{-} \mathrm{CD} 45 \mathrm{RB}^{\text {hi }}$ cells from $\mathrm{WT}$ CD 45.1 donors and Tregs $\left(\mathrm{CD} 4^{+} \mathrm{C} 25^{\text {hi }}\right)$ from either WT or Cd28- $\Delta$ Treg CD45.2 ani- mals. Mice receiving $C d 28-\Delta$ Treg transfer alone remained healthy (Figure 8B). This is consistent with the data in Cre-heterozygous mice, where $C d 28-\Delta$ Tregs did not induce disease, although that "assay" occurred in the presence of WT Tregs (Figure 6). These colitis data further demonstrate a lack of inherent pathogenicity in $C d 28-\Delta$ Tregs. However, while $C d 28-\Delta$ Tregs were not pathogenic, they failed to fully suppress colitis induced by naive $T$ cells, though the weight loss of mice was slightly delayed compared with those receiving naive $T$ cells alone (Figure $8 \mathrm{~B}$ ). Mice receiving naive $\mathrm{T}$ cells plus $C d 28-\Delta$ Tregs had obvious histologic evidence of severe colitis with mononuclear cell infiltration through multiple layers of the bowel (Figure 8C). Consistent with these results, at the time of sacrifice, mice that received $C d 28-\Delta$ Tregs had much higher percentages of effector cells and lower frequencies of $\mathrm{FOXP}^{+}$cells in the colon (Figure 8, D and E) compared with mice receiving WT Tregs.

One of the disadvantages of the adoptive transfer colitis model is the potentially confounding issues of homeostatic expansion, cell homing, and cell survival (23). Indeed, part of the reason for the failure of $C d 28-\Delta$ Tregs to suppress colitis may be their relative lack of homeostatic expansion in the immunodeficient environment of that model (Figure $8 \mathrm{~F}$ ). In order to examine the characteristics and function of $C d 28-\Delta$ Tregs under more physiologic conditions, we first grafted BALB/c skin onto female $C d 28^{f l / f l}$ Foxp $3^{Y F P \text {-Cre/+ }}$ mice. We analyzed the percentage of CD28-sufficient and CD28-deleted Tregs present (in the same animals) in peripheral blood before transplant compared with the percentage 8 days after transplant (Figure 9, A and B). Blood was chosen to study, as it enabled us to sample the same animals before and after transplant. We found only a small increase in CD28-deficient Tregs in response to the skin transplant, while in contrast the percentage of CD28-sufficient Tregs doubled.

To study Treg function, we used an induced model of experimental allergic encephalomyelitis (EAE), in which disease resolution is Treg dependent (24). We observed equivalent kinetics, incidence, and disease score in WT and Cd28- $\Delta$ Treg mice (Figure $9 \mathrm{C}$ and data not shown). Strikingly, however, disease began to resolve within about 10 to 15 days after onset in WT mice, while it continued to progress in $C d 28-\Delta$ Treg mice, necessitating sacrifice of the animals (Figure 9C). Together, these two models show a requirement for CD28 expression on Tregs for expansion/accumulation of cells and for appropriate resolution of inflammation. 

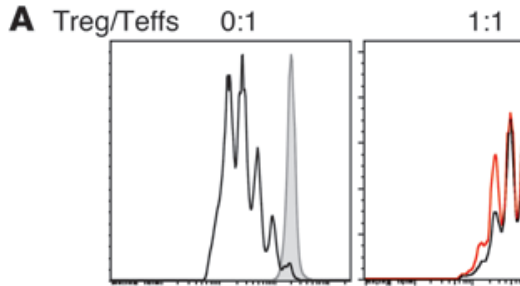

$1: 3$

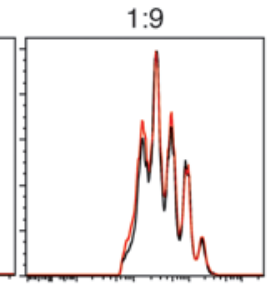

- WT Treg
- Cd28-sTre

Cell trace

B

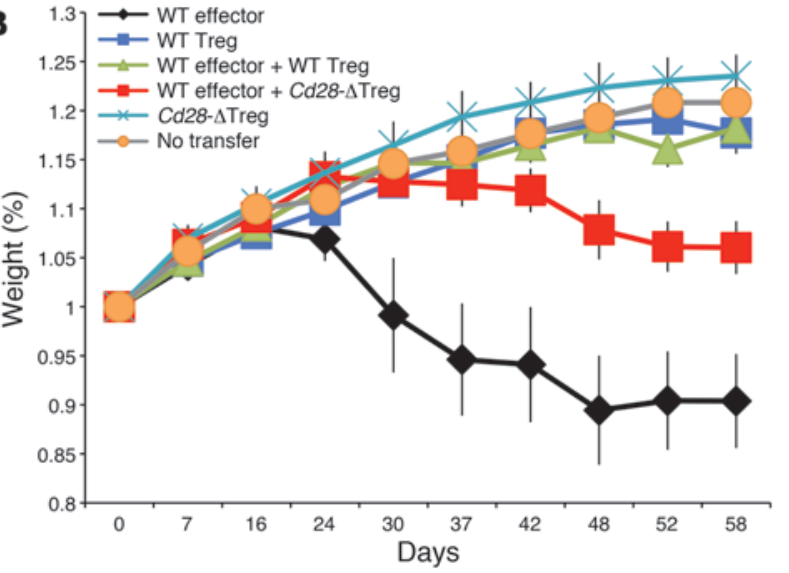

C

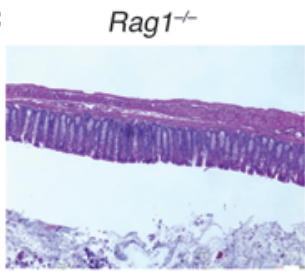

Teffs + WT Tregs

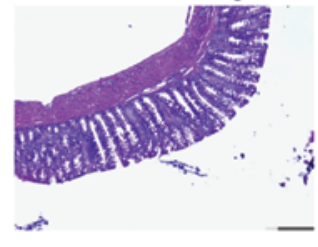

Teffs

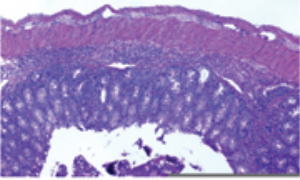

Teffs $+C d 28-\Delta$ Tregs

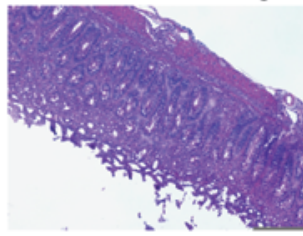

D

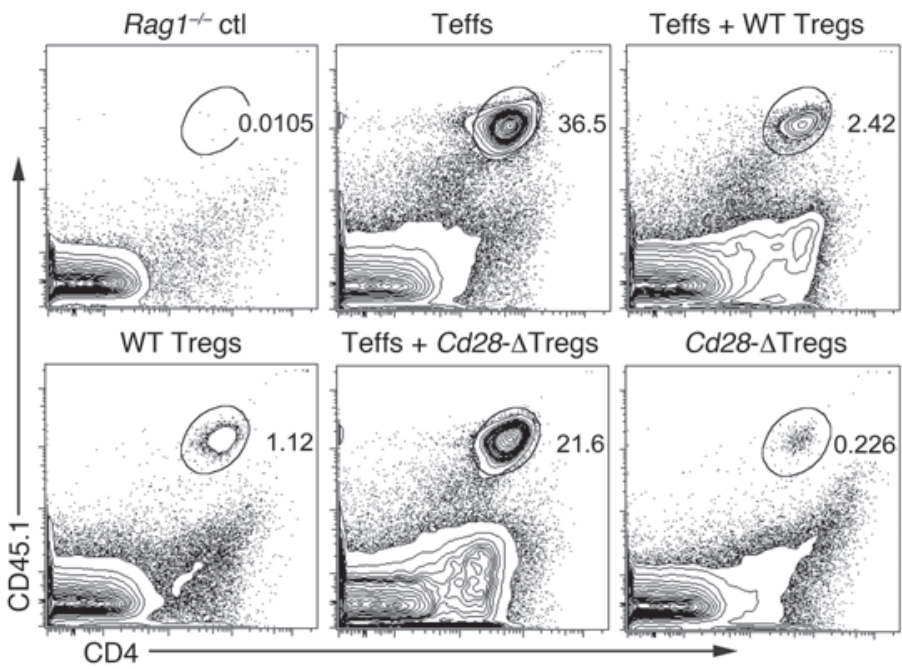

E

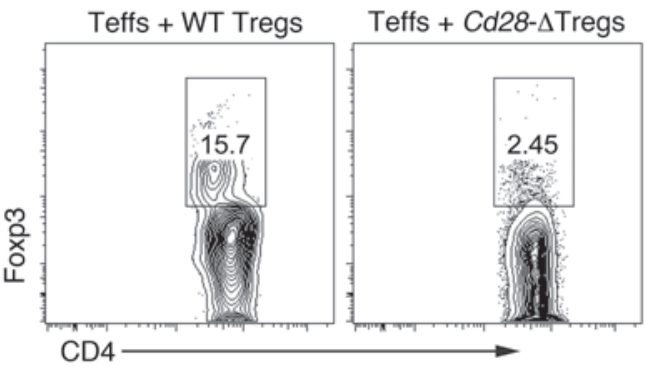

$\mathbf{F}$

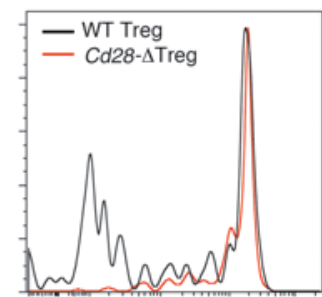

Cell tracer

Figure 8

Suppressive function of Cd28- $\Delta$ Tregs. (A) In vitro suppression assay. Sorted WT naive cells were stimulated by soluble CD3 and T cell-depleted splenocytes with the addition of different ratios of WT Tregs or Cd28- $\Delta$ Tregs. Tregs were sorted from 4-week-old mice. Teff, effector T cells. (B) In vivo colitis induction. Weight loss of Rag $1^{-1-}$ mice adoptively transferred with sorted CD4+CD45RB ${ }^{\text {hi }}$ effector $\mathrm{T}$ cells with or without WT Tregs or Cd28- $\Delta$ Tregs. A total of 6 mice were analyzed for each group. Data represent mean \pm SEM. (C) H\&E staining of the colons of mice in B. Original magnification, $\times 100$. (D and E) Representative analysis of donor CD45.1+CD4+ effector cells and regulatory cells in the colons of mice in $\mathbf{B}$. (F) Sorted WT Tregs or Cd28- $\Delta$ Tregs were labeled with CellTrace Violet and adoptively transferred to Rag $1^{-/-}$hosts. Lymph node cells were analyzed 7 days after transfer. Data are representative of 3 experiments. 

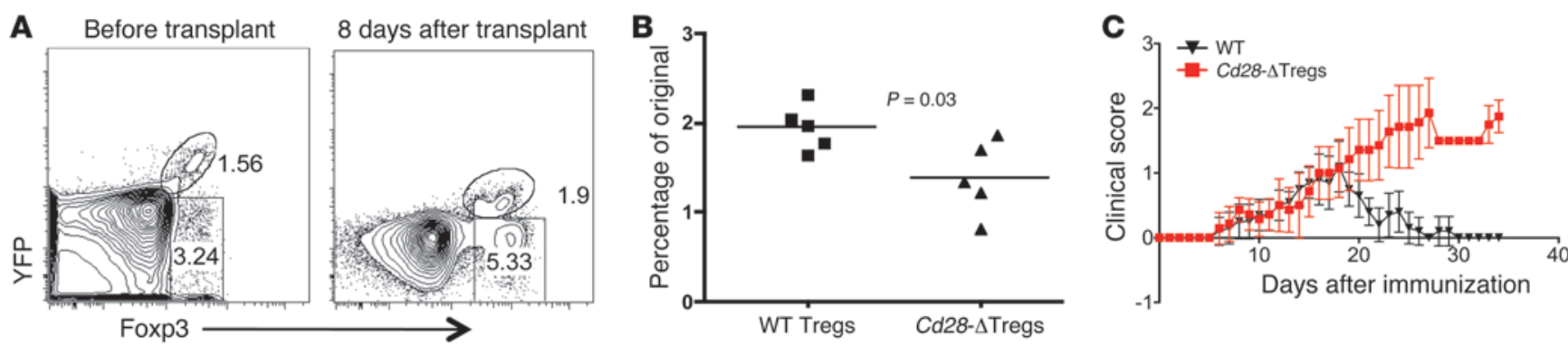

\section{Figure 9}

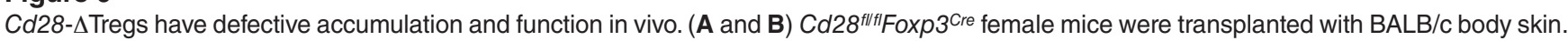
WT and Cd28- $\Delta$ Tregs from the blood were analyzed before or 8 days after transplantation. The percentage of increase of Tregs was analyzed in B, and each dot represents 1 mouse (horizontal bars indicate the mean). (C) Clinical scores of rMOG/CFA plus pertussis toxin-induced EAE in female WT Cd28 $8^{+/+} \times$FOXP3 $3^{\text {YFP-Cre/YFP-Cre }}(\mathrm{WT})$ or Cd28 $8^{\text {flft }} \times$ FOXP3 YFP-Cre/YFP-Cre $(C d 28-\Delta$ Treg) mice. A total of 7 mice in each group are shown. Data represent mean \pm SEM.

Cd28- $\Delta$ Tregs have reduced expression of CTLA-4, PD-1, and CCR6. The data above indicated a defect in $C d 28-\Delta$ Treg-mediated suppression. Tregs may use multiple mechanisms to maintain immune surveillance in vivo. CTLA-4 has been shown to be important for Treg function $(16,17)$, and PD-1/PDL-1 interactions have been implicated as well $(25,26)$. We found a roughly $50 \%$ reduction of CTLA- 4 and PD- 1 expression in Cd28- $\Delta$ Tregs (Figure 10, A and B). This was not a global reduction of Treg signature molecules, as the expression of FOXP3, CD25, and GITR was normal. Moreover, this was a cell-intrinsic process, as shown by examination of Tregs from female $C d 28^{f / f l} \mathrm{Foxp} 3^{Y F P-C r e /+}$ mice, where comparison of $\mathrm{YFP}^{+}$(i.e., CD28-deficient) cells with YFP- (i.e., CD28-sufficient) cells within the same animals revealed defects in expression of CD25, CD44, CTLA-4, PD-1, GITR, and FOXP3 (Figure 10D). Tregs are known to upregulate CTLA-4, PD-1, and other activation markers upon stimulation (27-30). To determine how loss of CD28 affected this process we stimulated cells in vitro with anti-CD3 plus APCs for 3 days. As shown in Figure 10E, and consistent with the data for cells directly ex vivo (Figure 10, A and B), we observed prominent differences in upregulation of CD44 and PD-1 and, reproducible, but smaller differences in upregulation of CTLA-4, CD25, and CD69 in Cd28- $\Delta$ Tregs.

As with conventional T cells, Tregs can circulate between peripheral and lymphoid tissues (28), and multiple chemokine receptors direct Treg migration $(31,32)$. As shown in Figure 10C, Cd28$\Delta$ Tregs directly ex vivo expressed reduced levels of CCR6, while other chemokine receptors, such as CCR4, CCR7, and CXCR4, were normal compared with WT Tregs. CCR6 is involved in the migration of Tregs to inflammatory tissues (33). The failure of upregulation of CCR 6 in $C d 28-\Delta$ Tregs is consistent with the observation of reduced $\mathrm{FOXP}^{+}$cells in the skin of $C d 28-\Delta$ Treg mice (Figure 5). Collectively, these data suggest that defects in activation induced Treg markers and failure to migrate into inflammatory sites may contribute to the inability of these cells to adequately regulate immune responses in vivo.

\section{Discussion}

Previous studies have yielded conflicting results as to whether or not CD28 signaling is required for Treg function. Tai et al. found that $\mathrm{Cd} 28^{-/-} \mathrm{CD} 4^{+} \mathrm{CD} 25^{+}$thymocytes were unable to suppress in vitro; however, the requirement of CD28 for Treg development suggests that these were not a priori "imprinted" nTregs (15). Lyd- dane and colleagues as well as Guo et al. reported that CD28 is required for iTreg generation but not function $(34,35)$. However, earlier work has not been able to address the issue of the role of CD28 in the function, maintenance, or survival of thymic-derived Tregs. To address this issue, we have created $C d 28-\Delta$ Treg mice in which CD28 is deleted under control of the FOXP3 promoter.

While Treg numbers are preserved in $C d 28-\Delta$ Treg mice, they nonetheless develop a systemic autoimmune process, most prominently affecting the liver and the skin. These inflammatory processes are reminiscent of the phenotype observed in scurfy mice (albeit less severe), consistent with a loss of suppressive functions in $C d 28-\Delta$ Tregs and their failure to maintain immune surveillance in vivo. This is in contrast to their ability to suppress proliferative responses in an in vitro suppression assay. Such discordance between in vitro and in vivo functions of Tregs is being reported with increased frequency in various models and clearly reflects the complexity and multifaceted nature of the in vivo response.

The most striking abnormalities that we observed in $\mathrm{Cd} 28$ $\Delta$ Tregs were marked decreases in CTLA-4, PD-1, and CCR6. CTLA-4 is upregulated by CD28 costimulatory engagement in non-Tregs (36) and also is required for Treg suppressive function $(16,17)$. PD-1 and PDL-1 signaling have been shown to regulate the development of induced Tregs (25) but have not been linked previously to alternations in Treg function. CCR6 recently was linked to the ability of Tregs to home to inflammatory sites, and thus decrease in expression of this molecule may be part of the spectrum of impaired regulation in $C d 28-\Delta$ Treg mice. In contrast to the severe lesions in the skin and lungs, other tissues, most notably the small intestine and colon, were generally spared in $C d 28-\Delta$ Treg mice. Whether or not this is a result of differential usage of homing receptors for Tregs in the gut is not yet known.

In addition to the in vivo functional defect in suppression in Cd28- $\Delta$ Tregs, we observed a separable defect in homeostasis, which was only detected in a competitive or a lymphopenic environment. Thus, over time, in mixed bone marrow chimeras and female $C d 28^{f / f l}$ mice heterozygous for Foxp $3^{\mathrm{Cre}}, \mathrm{Cd} 28-\Delta$ Tregs almost disappeared and the Treg compartment was comprised almost completely of CD28-sufficient cells. Studies in the female heterozygotes indicated that this was accompanied by a defect in the homeostatic proliferation of $C d 28-\Delta$ Tregs. It is likely, however, that $C d 28-\Delta$ Tregs have a survival defect as well. When such cells were transferred alone, we noted a defect in lymphopenia-induced expansion (Figure 8F). 
A

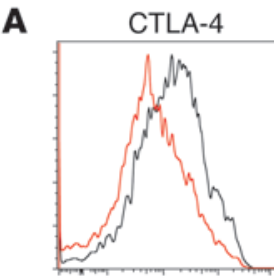

MFI
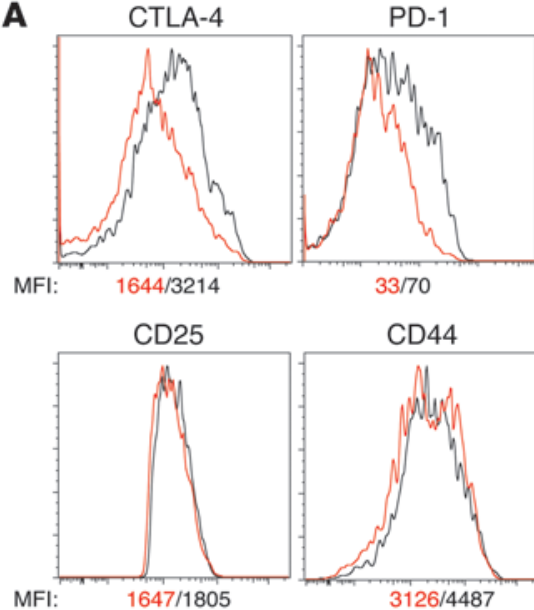

$33 / 70$
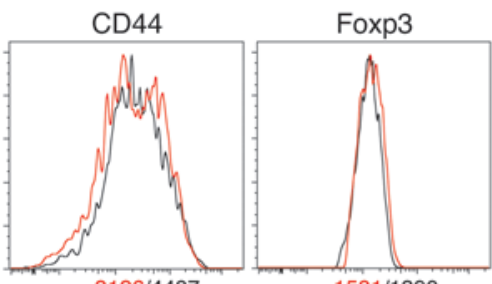

$1581 / 1390$

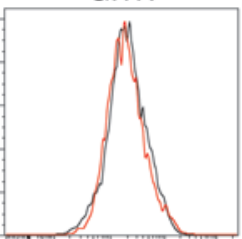

$2780 / 2829$

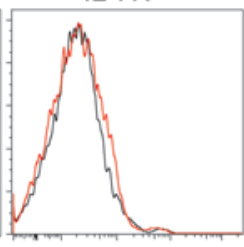

$351 / 340$

C

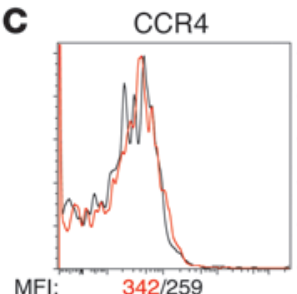

CCR6
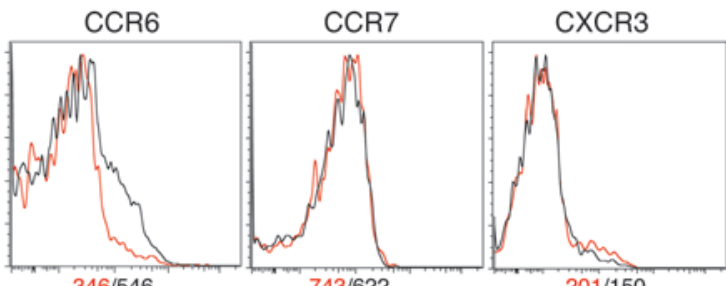

D

Foxp3

CTLA-4

PD-1

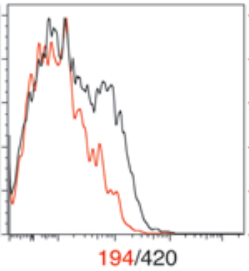

CD103

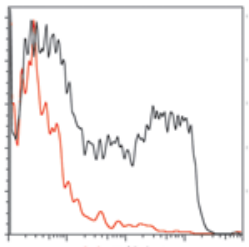

2798/5961

$36.5 / 207$

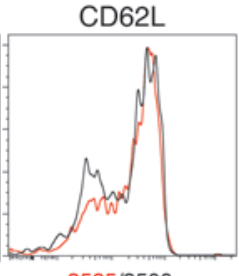

$3585 / 3500$

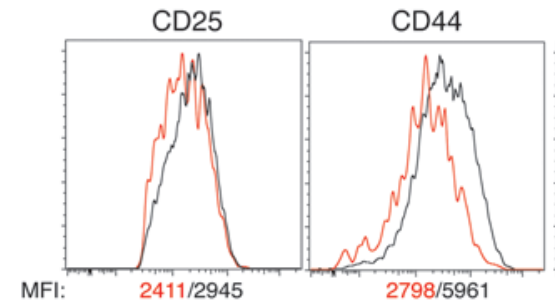

CD44

E

E $\quad \mathrm{CD} 25$

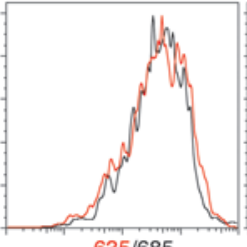

MFI:

635/685

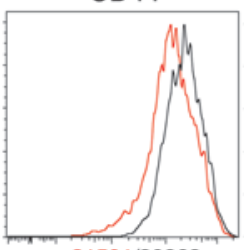

21501/30862

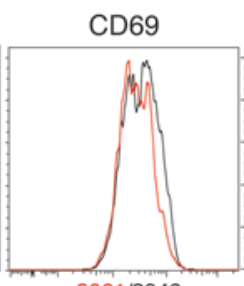

$3061 / 3948$

PD-1

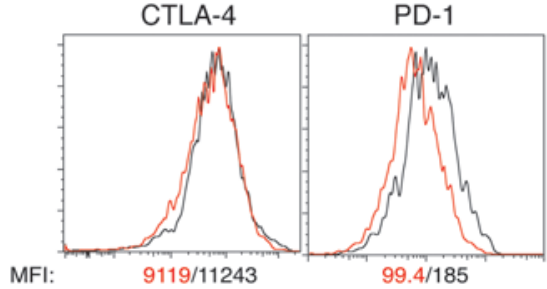

B

CTLA-4

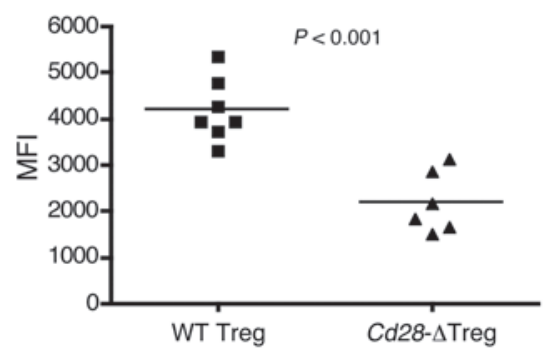

PD-1

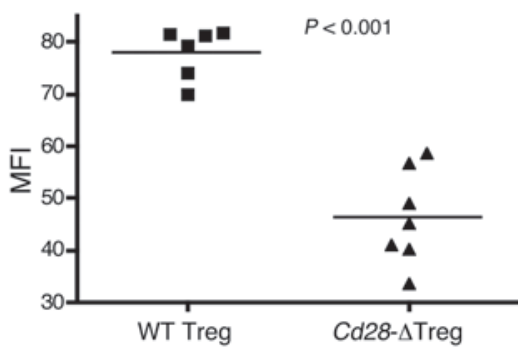

WT Tregs

Cd28- $\Delta$ Tregs

\section{Figure 10}

Cell surface markers in Cd28- $\Delta$ Tregs. (A-C) Analysis of $\mathrm{CD} 4{ }^{+} \mathrm{CD} 25^{+} \mathrm{YFP}+$ cells from lymph

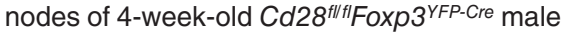
mice. Symbols represent individual mice; horizontal bars indicate the mean. (D) Comparison of WT and Cd28- $\Delta$ Tregs in a representative

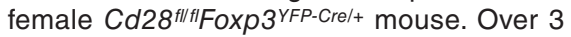
mice were analyzed. (E) Activation markers in sorted WT Tregs or Cd28- $\Delta$ Tregs stimulated for 3 days with soluble CD3 plus mitomycin-treated T cell-depleted splenocytes. Experiments were repeated 3 times. 
Moreover, in the adoptive transfer colitis studies, very few $C d 28$ $\Delta$ Tregs could be recovered from mice receiving these cells, either alone or with WT effectors, and it is likely that their defective survival in this latter model is a major contributor to failure of colitis prevention. It is interesting to note that colitis was not an observed pathologic feature of the autoimmune syndrome that the $C d 28$ $\Delta$ Treg animals developed. There are a number of potential explanations for this, including the effects of homeostatic expansion, alterations in cell migration, lack of new thymic Tregs being generated in the colitis model, differences in gut flora in between immunocompetent and immunodeficient animals, etc.

Interestingly, the only tissue in which we observed decreased numbers of Tregs was within the thymus. CD28 has a cell-intrinsic role in thymic Treg generation via its Lck-binding motif, and this function is independent of the ability of this motif to promote IL-2 production (15). As CD28 is not deleted until after FOXP3 is expressed, this result suggests an intrathymic role for CD28, either in the maintenance of FOXP3 expression or the proliferation/survival of thymic FOXP3 ${ }^{+}$cells. The decrease in BrdU incorporation in Tregs lacking CD28 (or even those which are haplosufficient for CD28; Figure 3, A and B) argues for the latter possibility.

Recently, two groups found that a GFP-FOXP3 fusion protein, commonly used to track Tregs, was actually a hypomorph with altered molecular interactions and impaired downstream activation of the normal Foxp3 gene expression/repression program (37, 38). In contrast, in the mice that we used, YFP-Cre is driven by an IRES, and thus the FOXP3 protein is intact and unaltered. Indeed, in female $C d 28^{f l /+} \mathrm{Foxp} 3^{\mathrm{YFPCre} /+}$ mice, we see similar percentages of $\mathrm{YFP}^{+}\left(\mathrm{CD}^{2} 8^{-}\right)$and $\mathrm{YFP}^{-}\left(\mathrm{CD} 28^{+}\right)$Tregs $(2.84 \pm 0.43$ vs. $2.5 \pm 0.54$; $n=4)$, consistent with the fact that the Foxp $3^{\text {Cre }}$ genotype does not intrinsically impair Treg survival.

One potential concern regarding the use of Foxp $3^{\text {Cre }}$ as a means to target Tregs is the fact that FOXP3 may be transiently expressed by cells that are not truly committed to the Treg "lineage." Recent studies have yielded conflicting results on the stability of FOXP3 expression and the question of whether or not there is a substantial population of ex-FOXP3 cells that have "diverted" into a Th lineage. We believe that this issue is unlikely to bear upon the interpretation of our data for at least two reasons. First, in mice CD28 is normally expressed in all $\mathrm{T}$ cells (39). In $C d 28-\Delta$ Treg mice, we did not detect a significant population of CD28-FOXP3- (i.e., CD28-YFP-) cells, suggesting that $\mathrm{CD} 28$ deletion is confined to cells that continue to express FOXP3. Given the role of CD28 in cell survival, it is likely that cells that are not Tregs, but transiently express FOXP3 and delete CD28, find themselves at a competitive disadvantage and are rapidly lost. Second, even if a population of "ex-FOXP3" effector/ memory cells was present, they are extremely unlikely to account for the autoimmune pathology observed in $C d 28-\Delta$ Treg mice, as they would have been expected to cause disease in the mixed bone marrow chimeras and female Cre-heterozygous mice.

In summary, our data reveal distinct Treg-intrinsic roles for CD28 in both regulatory function and survival. These studies provide an additional mechanism whereby blockade of the ligands for CD28 and CTLA-4, namely CD80 and CD86, may in some circumstances enhance immune responses and contribute to acceleration of autoimmune processes or transplant rejection.

\section{Methods}

Gene targeting and generation of $C d 28^{f / f l}$ mice. A genomic $C d 28$ fragment from BAC clone no. RP2478J76 (CHORI) and vectors PL253, PL451, and PL452 were used for preparation of a targeting vector to insert 2 LoxP sites, which would flank exon 2 and exon 3 of murine $C d 28$ (Figure 1A). Detailed methodology of the recombineering protocol can be found at http:// ncifrederick.cancer.gov/research/brb/protocol.aspx. Briefly, 1 LoxP sequence was inserted $150 \mathrm{bp}$ upstream of exon 2, and the other LoxP sequence was placed downstream of exon 3 along with a neomycin selection cassette. An approximately 10-kb floxed CD28 gene fragment was retrieved from the BAC and placed in a PL253 targeting vector. This construct was linearized and electroporated into the B6 ES cell line PB6, and G418-resistant colonies were selected. The $5^{\prime}$ LoxP fragment introduced a new EcoRV site, and thus the floxed allele gave rise to a smaller $10-\mathrm{kb}$ fragment ( $3^{\prime}$ probe) or a $6-\mathrm{kb}$ fragment $\left(5^{\prime}\right.$ probe) as compared with the 14-kb fragment in the WT allele by Southern blot after EcoRV digestion (Figure 1B). We obtained 12 positive ES clones that were confirmed by Southern blotting. Three clones were injected into pseudopregnant female mice at the Penn Gene Targeting Core (University of Pennsylvania). Chimeric mice born from $2 \mathrm{ES}$ clones exhibited germline transmission to their pups, which were intercrossed to produce $C d 28^{f / / f l}$ mice. These mice then bred with Foxp3 ${ }^{\text {YFP-Cre }}$ transgenic mice (a gift from A. Rudensky, Memorial Sloan-Kettering Cancer Center, New York, New York, USA) to generate mice with a deletion of $\mathrm{CD} 28$ specific to the $\mathrm{T}$ regulatory cell compartment. Mice were genotyped by PCR of tail DNA. Offspring that were found to express the Foxp $3^{\text {YFP-Cre }}$ transgene and were homozygous for the CD28floxed allele $\left(C d 28^{f / f t}\right)$ were used for analysis as homozygous mutant mice (Cd28- $\Delta$ Treg), while $C d 28^{+/+}$Foxp $3^{\text {YFP-Cre }}$ animals were used as littermate controls. All colonies were maintained under specific pathogen-free conditions at the animal facilities of Beth Israel Deaconess Medical Center. Rag1 1/-, CD 45.1 $1^{+}$, and Thy $1.1^{+} \mathrm{B} 6$ mice were purchased from The Jackson Laboratory, and B7-1/B7-2 double-knockout mice (B6 background) were a gift of Arlene Sharpe (Harvard Medical School).

Western blotting. T cells were lysed in ice-cold RIPA lysis buffer (SigmaAldrich) with protease and phosphatase inhibitors. Nuclei were removed by high-speed microcentrifugation in the cold, and samples were diluted 2:1 in $2 \times$ Laemmli sample buffer before gel loading. Anti-CD28 (c-20, Santa Cruz Biotechnology Inc.) was used to detect CD28 expression.

Media, reagents, antibodies, and flow cytometry. Cells were grown in RPMI 1640 (Mediatech Inc.) supplemented with 10\% heat-inactivated FBS, 100 $\mathrm{U} / \mathrm{ml}$ penicillin, $100 \mathrm{mg} / \mathrm{ml}$ streptomycin, $2 \mathrm{mM}$ L-glutamine, and $50 \mathrm{mM}$ 2-mercaptoethanol (Sigma-Aldrich). Fluorescent anti-CD4, anti-CD25, anti-CD44, anti-CD62L, anti-CD8, anti-CD103, anti-CCR4, anti-CCR6, and anti-CCR7 antibodies were purchased from BioLegend. Anti-CTLA-4, anti-PD-1, anti-GITR, anti-CD127, anti-IFN, anti-CXCR3, and antiCXCR4 were purchased from BD Biosciences - Pharmingen. The antiFOXP3 Staining Kit was purchased from eBioscience. Murine recombinant IL-2 (rIL-2) was purchased from R\&D Systems. BD Cytofix/Perm buffer was used for intracellular staining. Cells were analyzed on a BD FACSCalibur or a BD LSR II (BD).

Naive and Treg isolation. Spleen and lymph node cells were isolated from 4- to 8-week-old mice and enriched for $\mathrm{CD}^{+}$cells with the mouse $\mathrm{CD} 4^{+}$ Enrichment Kit (Stemcell Technologies). Cells were stained with antiCD25 PE, CD44-PerCP-cy5.5, CD62L-APC, and CD4-APC-cy7. CD4+ $\mathrm{CD} 25^{-} \mathrm{YFP}^{-} \mathrm{CD} 44^{\mathrm{lo}} \mathrm{CD} 62 \mathrm{~L}^{\text {hi }}$ naive cells and $\mathrm{CD} 4^{+} \mathrm{CD} 25^{+} \mathrm{YFP}^{+}$Tregs were sorted by flow cytometry on a FACSAria Cell Sorter (BD Biosciences). Cell purity was routinely greater than $95 \%$. In an in vivo colitis model, CD 45.1 B6 mice were used to sort $\mathrm{CD} 4^{+} \mathrm{CD} 25^{-} \mathrm{CD} 45 \mathrm{RB}^{\text {hi }}$ effector T cells.

Cell activation and proliferation assays. For total splenocyte stimulation, $1 \times 10^{6}$ splenocytes were stimulated by Leukocyte Activation Cocktail (BD Biosciences) for 6 hours and then fixed for cytokine staining.

For Treg proliferation assays, FACS-sorted $\mathrm{CD}^{+}{ }^{+} \mathrm{CD} 25^{+} \mathrm{YFP}^{+}$cells were labeled with CellTrace Violet and cultured in complete medium in the pres- 
ence of mitomycin-treated T cell-depleted splenocytes in 96-well plates at a density of $10^{5}$ cells per well. For TCR stimulation, cells were cultured with soluble $0.5 \mu \mathrm{g} / \mathrm{ml}$ anti-CD3 $(2 \mathrm{C} 11, \mathrm{BD})$ supplemented with or without 10 $\mathrm{ng} / \mathrm{ml}$ rIL-2. Cell division was analyzed by CellTrace Violet dilution after 72 hours. For assessment of in vivo proliferation, CellTrace Violet-labeled Tregs were adoptively transferred to Rag $1^{1^{-/}}$mice by i.v. injection, and mice were sacrificed after 7 days. In all instances, cell viability was determined by flow cytometry using Live/Dead Fixable Aqua (Invitrogen).

In vitro suppression assays. $1.5 \times 10^{5} \mathrm{CD} 4^{+} \mathrm{CD} 25^{-} \mathrm{CD} 44^{\mathrm{lo}} \mathrm{CD} 62 \mathrm{~L}^{\text {hi }} \mathrm{T}$ cells were labeled by CellTrace Violet (Invitrogen) and cultured with $4.5 \times 10^{5}$ irradiated T cell-depleted splenocytes plus $0.5 \mu \mathrm{g} / \mathrm{ml}$ anti-CD3. The indicated ratios of $\mathrm{CD} 4{ }^{+} \mathrm{CD} 25^{+} \mathrm{YFP}^{+}$cells from either WT or $C d 28-\Delta$ Treg mice were added to the cultures. CellTrace Violet profiles on YFP- cells were assessed by flow cytometry after 72 hours.

$T$ cell differentiation assays. Sorted Tregs $\left(\mathrm{CD}^{+} \mathrm{YFP}^{+}\right)$or naive $\mathrm{T}$ cells $\left(\mathrm{CD} 4^{+} \mathrm{YFP}^{-} \mathrm{CD} 44^{\mathrm{lo}}\right)$ were cultured in complete medium in the presence of mitomycin-treated T cell-depleted splenocytes in 96-well plates at a density of $10^{5}$ cells per well. $1 \mu \mathrm{g} / \mathrm{ml}$ soluble CD3 was added as the stimuli. For Th1 differentiation, mouse rIL-2 (10 ng/ml), IL-12 $(5 \mathrm{ng} / \mathrm{ml})$, and anti-IL-4 $(10 \mu \mathrm{g} / \mathrm{ml})$ were added. For Th17 differentiation, IL-1 (10 ng/ml), IL-6 (50 $\mathrm{ng} / \mathrm{ml})$, TGF- $\beta 1(1 \mathrm{ng} / \mathrm{ml})$, anti-IFN- $\gamma(10 \mu \mathrm{g} / \mathrm{ml})$, and anti-IL-2 $(10 \mu \mathrm{g} / \mathrm{ml})$ were added into the culture. After 4 days of culture, cells were restimulated by PMA plus ionomycin with the presence of Golgi block for determination of cytokine production.

In vivo BrdU labeling. Mice were injected i.p. with $1 \mathrm{mg} \mathrm{BrdU}$ (BD Biosciences) every 12 hours for 3 days. Mice were sacrificed 12 hours after the final injection, and $2 \times 10^{6}$ lymphocytes, splenocytes, or thymocytes were surface stained for CD4, CD8, CD25, and FOXP3. BrdU staining was performed using a BrdU Labeling Kit (BD Biosciences) per the manufacturer's instructions.

Histology. Tissues were harvested and fixed overnight at $4^{\circ} \mathrm{C}$ in $10 \%$ neutral buffered formalin. Following fixation, tissues were rinsed with cold PBS and embedded in paraffin. Tissues were sectioned $(5 \mu \mathrm{m})$ and examined by H\&E staining. For immunohistochemical analysis, skin sections were incubated with antibodies against mouse CD4, CD8, IL-17, IFN- $\gamma$, and Gr-1 (BD Bioscience); CD25 and FOXP3 (BioLegend); and F4/80 (AbD Serotec), followed by biotinylated secondary antibodies (Vector). Sectioning and staining were performed by the histology core at Beth Israel Deaconess Medical Center.

Bone marrow chimeras. C57BL/6 Thy1.1 mice were irradiated (10 Gy) prior to reconstitution with $4 \times 10^{6}$ total bone marrow cells from WT (CD45.1Thy1.2) and $C d 28-\Delta$ Treg (CD45.2Thy1.2) mice at 1:1 ratio. Five months later, peripheral blood mononuclear cells were analyzed flow cytometry.

In vivo colitis model. Rag $1^{-/-}$mice were injected i.v. with $6 \times 10^{5} \mathrm{CD} 4^{+} \mathrm{CD} 25^{-}$ CD45RB hi T cells, either alone or with $2 \times 10^{5} \mathrm{WT}\left(\mathrm{CD}^{+} \mathrm{CD} 25^{+}\right)$Tregs or

$C d 28-\Delta$ Tregs $\left(\mathrm{CD}^{+}{ }^{+} \mathrm{CD} 25^{+} \mathrm{YFP}^{+}\right)$. Mice were weighed and examined every week for signs of disease and euthanized for tissue harvest at 8 to 10 weeks. Cells from lymph nodes, spleens, and colons were isolated and stained for CD4, CD25, and FOXP3. Colon tissues were fixed in 10\% neutral buffered formalin (Fisher Scientific), cut into 5-mm sections, and stained with H\&E. Skin transplantation. Recipient mice were anesthetized with ketamine and xylazine, and a 1- $\times 1-\mathrm{cm}$ area of dermis was removed from the lateral trunk. A full-thickness donor skin graft was sutured to the exposed s.c. tissue bed using 4.0 chromic absorbable suture, and animals were bandaged after application of antibiotic ointment to the graft.

EAE. Animals were immunized s.c. in the abdominal flank with $200 \mu \mathrm{l}$ myelin oligodendrocyte glycoprotein peptide $\left(\mathrm{MOG}_{35-55}\right)$ (UCLA Biopolymers Core) emulsified in complete Freund's adjuvant (Sigma-Aldrich), followed by i.p. injection of $200 \mu \mathrm{l}$ pertussis toxin (List Biological Laboratories) on days 0 and 2 after immunization. Immunized animals were monitored daily for weight loss and clinical disease score in a blinded manner. Disease was scored as follows: 0 , no disease; 1 , flaccid tail paralysis; 2 , hind limb paresis; 3, bilateral hind limb paralysis; 4 , hind and fore limb paralysis.

Statistics. Comparison of means between groups was done by using the 2 -tailed Student's $t$ test. Differences were considered statistically significant at $P<0.01$.

Study approval. All experiments described in this manuscript were approved by the Institutional Animal Care and Use Committee at the Beth Israel Deaconess Medical Center or the University of Pennsylvania.

\section{Acknowledgments}

We thank Terry Strom, Arlene Sharpe, Christophe Benoist, and members of the Turka, Strom, and Maltzman laboratories for many helpful discussions and Eva Csizmadia and Weihua Gong for technical assistance. This work was funded by NIH grants AI-037691 and AI-085160.

Received for publication May 25, 2012, and accepted in revised form November 1, 2012.

Address correspondence to: Laurence A. Turka, Transplantation Biology Research Center, Massachusetts General Hospital, MGHEast; Building 149-9019, 13th Street, Boston, Massachusetts 02129, USA. Phone: 617.724.7740; E-mail: lturka@partners.org.

Laurence A. Turka, Ruan Zhang, Alexandria Huynh, and JiHoon Chang's present address is: Department of Surgery, Massachusetts General Hospital and Harvard Medical School, Boston, Massachusetts, USA.

1. Khattri R, Cox T, Yasayko SA, Ramsdell F. An essential role for Scurfin in CD4(+)CD25(+) T regulatory cells. Nat Immunol. 2003;4(4):337-342.

2. Fontenot JD, Gavin MA, Rudensky AY. Foxp3 programs the development and function of CD4(+) CD25(+) regulatory T cells. Nat Immunol. 2003; 4(4):330-336.

3. Hori S, Nomura T, Sakaguchi S. Control of regulatory $\mathrm{T}$ cell development by the transcription factor Foxp3. Science. 2003;299(5609):1057-1061.

4. Wildin RS, Smyk-Pearson S, Filipovich AH. Clinical and molecular features of the immunodysregulation, polyendocrinopathy, enteropathy, $\mathrm{X}$ linked (IPEX) syndrome. J Med Genet. 2002;39(8):537-545.

5. Godfrey VL, Wilkinson JE, Russell LB. X-linked lymphoreticular disease in the scurfy (sf) mutant mouse. Am J Pathol. 1991;138(6):1379-1387.

6. Kim JM, Rasmussen JP, Rudensky AY. Regulatory T cells prevent catastrophic autoimmunity throughout the lifespan of mice. Nat Immunol. 2007;
8(2):191-197.

7. June CH, Bluestone JA, Nadler LM, Thompson CB. The B7 and CD28 receptor families. Immunology Today. 1994;15(7):321-331.

8. Bour-Jordan H, Esensten JH, Martinez-Llordella M, Penaranda C, Stumpf M, Bluestone JA. Intrinsic and extrinsic control of peripheral T-cell tolerance by costimulatory molecules of the CD28/ B7 family. Immunol Rev. 2011;241(1):180-205.

9. Wells AD, et al. Requirement for T-cell apoptosis in the induction of peripheral transplantation tolerance. Nat Med. 1999;5(11):1303-1307.

10. Li XC, Strom TB, Turka LA, Wells AD. T cell death and transplantation tolerance. Immunity. 2001; 14(4):407-416.

11. Salomon B, et al. B7/CD28 costimulation is essential for the homeostasis of the CD4+CD25+ immunoregulatory $\mathrm{T}$ cells that control autoimmune diabetes. Immunity. 2000;12(4):431-440.

12. Tang Q, et al. Cutting edge: CD28 controls periph- eral homeostasis of CD4(+)CD25(+) regulatory T cells. J Immunol. 2003;171(7):3348-3352.

13. Riella LV, et al. Deleterious effect of CTLA4-Ig on a Treg-dependent transplant model. Am J Transplant. 2012;12(4):846-855.

14. Lenschow DJ, et al. CD28/B7 regulation of Th1 and Th2 subsets in the development of autoimmune diabetes. Immunity. 1996;5(3):285-293.

15. Tai X, Cowan M, Feigenbaum L, Singer A. CD28 costimulation of developing thymocytes induces Foxp 3 expression and regulatory $\mathrm{T}$ cell differentiation independently of interleukin 2. Nat Immunol. 2005;6(2):152-162.

16. Wing $\mathrm{K}$, et al. CTLA-4 control over Foxp3+ regulatory T cell function. Science. 2008;322(5899):271-275.

17. Friedline RH, et al. CD4+ regulatory T cells require CTLA-4 for the maintenance of systemic tolerance. JExp Med. 2009;206(2):421-434.

18. Vincenti F, et al. Three-year outcomes from BENEFIT, a randomized, active-controlled, parallel- 
group study in adult kidney transplant recipients. Am J Transplant. 2012;12(1):210-217.

19. Rubtsov YP, et al. Regulatory T cell-derived interleukin-10 limits inflammation at environmental interfaces. Immunity. 2008;28(4):546-558.

20. Fisson $S$, et al. Continuous activation of autoreactive CD4+ CD25+ regulatory T cells in the steady state. J Exp Med. 2003;198(5):737-746.

21. Masteller EL, Tang Q, Bluestone JA. Antigen-specific regulatory $\mathrm{T}$ cells--ex vivo expansion and therapeutic potential. Semin Immunol. 2006;18(2):103-110.

22. Jordan MS, et al. Thymic selection of CD4+CD25+ regulatory $\mathrm{T}$ cells induced by an agonist self-peptide. Nat Immunol. 2001;2(4):301-306.

23. Barthlott T, Kassiotis G, Stockinger B. T cell regulation as a side effect of homeostasis and competition. J Exp Med. 2003;197(4):451-460.

24. McGeachy MJ, Stephens LA, Anderton SM. Natural recovery and protection from autoimmune encephalomyelitis: contribution of CD4+CD25+ regulatory cells within the central nervous system. J Immunol. 2005;175(5):3025-3032.

25. Francisco LM, et al. PD-L1 regulates the development, maintenance, and function of induced regu- latory T cells. J Exp Med. 2009;206(13):3015-3029.

26. Francisco LM, Sage PT, Sharpe AH. The PD-1 pathway in tolerance and autoimmunity. Immunol Rev. 2010;236:219-242.

27. Zhang N, et al. Regulatory $\mathrm{T}$ cells sequentially migrate from inflamed tissues to draining lymph nodes to suppress the alloimmune response. Immunity. 2009;30(3):458-469.

28. Tomura $\mathrm{M}$, et al. Activated regulatory $\mathrm{T}$ cells are the major $\mathrm{T}$ cell type emigrating from the skin during a cutaneous immune response in mice. J Clin Invest. 2010;120(3):883-893.

29. Gupta S, et al. Allograft rejection is restrained by short-lived TIM-3+PD-1+Foxp3+ Tregs. J Clin Invest. 2012;122(7):2395-2404.

30. Cheng G, Yuan X, Tsai MS, Podack ER, Yu A, Malek TR. IL-2 receptor signaling is essential for the development of Klrg1+ terminally differentiated T regulatory cells. J Immunol. 2012;189(4):1780-1791.

31. Wei S, Kryczek I, Zou W. Regulatory T-cell compartmentalization and trafficking. Blood. 2006; 108(2):426-431.

32. Siegmund K, et al. Migration matters: regulatory T-cell compartmentalization determines suppres- sive activity in vivo. Blood. 2005;106(9):3097-3104

33. Yamazaki T, et al. CCR6 regulates the migration of inflammatory and regulatory T cells. J Immunol. 2008;181(12):8391-8401.

34. Lyddane C, Gajewska BU, Santos E, King PD, Furtado GC, Sadelain M. Cutting Edge: CD28 Controls dominant regulatory $\mathrm{T}$ cell activity during active immunization. J Immunol. 2006;176(6):3306-3310.

35. Guo F, Iclozan C, Suh WK, Anasetti C, Yu XZ. CD28 controls differentiation of regulatory $\mathrm{T}$ cells from naive CD4 T cells. JImmunol. 2008;181(4):2285-2291.

36. Lindsten T, et al. Characterization of CTLA-4 structure and expression on human T cells. J Immunol. 1993;151(7):3489-3499.

37. Bettini ML, et al. Loss of epigenetic modification driven by the Foxp 3 transcription factor leads to regulatory $\mathrm{T}$ cell insufficiency. Immunity. 2012; 36(5):717-730

38. Darce J, et al. An N-terminal mutation of the Foxp3 transcription factor alleviates arthritis but exacerbates diabetes. Immunity. 2012;36(5):731-741.

39. Gross JA, Callas E, Allison JP. Identification and distribution of the costimulatory receptor CD28 in the mouse. J Immunol. 1992;149(2):380-388. 